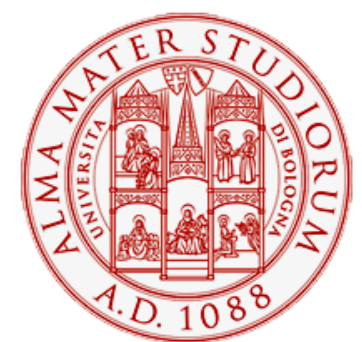

Alma Mater Studiorum - Università di Bologna DEPARTMENT OF ECONOMICS

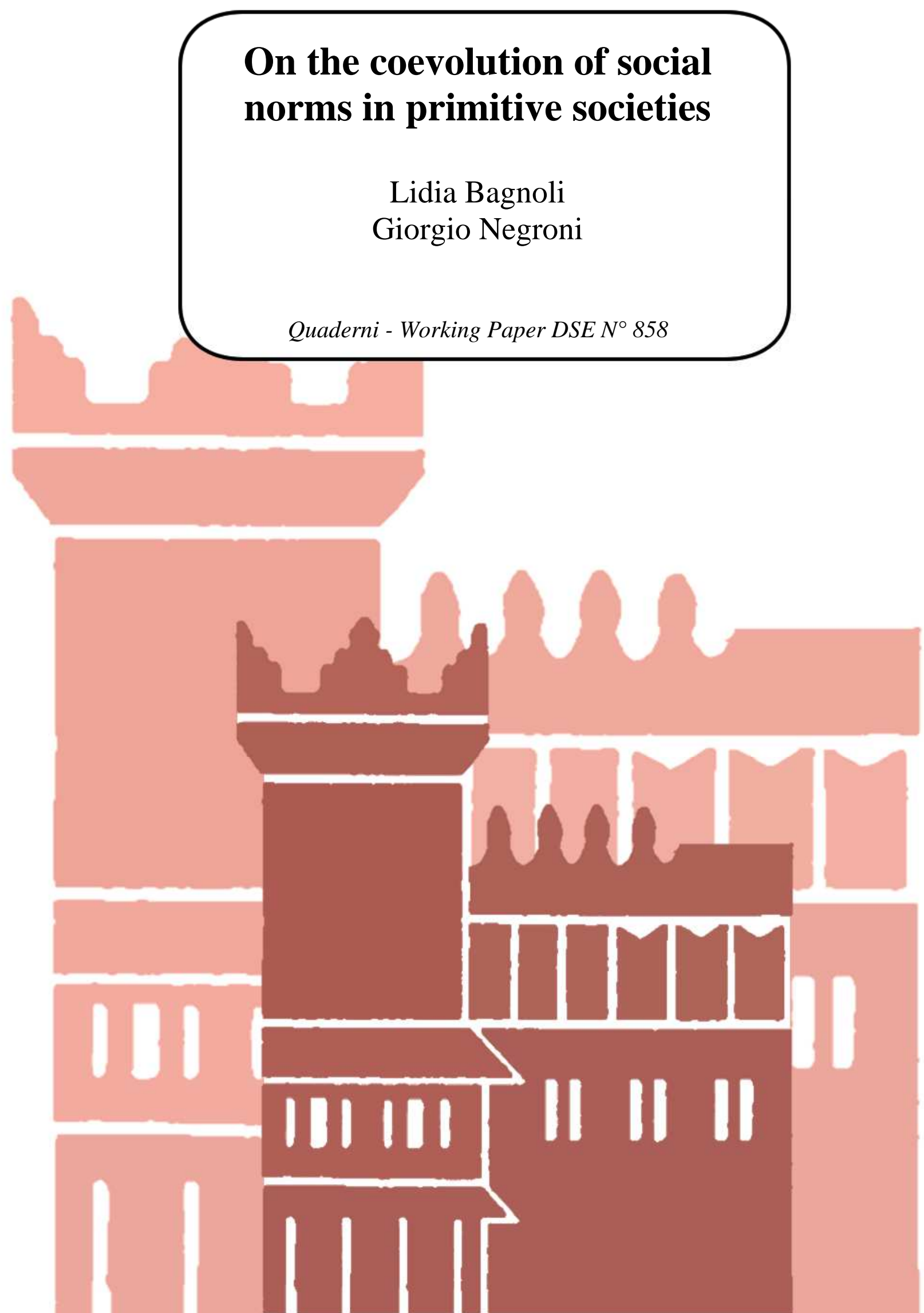




\title{
On the coevolution of social norms in primitive societies
}

\author{
Lidia Bagnoli ${ }^{1, a}$, Giorgio Negroni ${ }^{\dagger}, a$ \\ ${ }^{a}$ Department of Economics, University of Bologna, \\ Strada Maggiore 45, 40125 Bologna, Italy \\ E-mail: lidia.bagnoli@tesoro.it; giorgio.negroni@unibo.it.
}

\begin{abstract}
.
Two parties bargaining over a pie, the size of which is determined by their previous investment decisions. The bargaining rule is sensitive to investment behavior. Two games are considered. In both, bargaining proceeds according to the Nash Demand Game when a symmetric investments profile is observed. When, on the other hand, an asymmetric investments profile is observed, we assume that bargaining proceeds according to the Ultimatum Game in one case and according to a Dictator Game in the other. We hereby show that in both games when a unique stochastically stable outcome exists it supports an homogeneous behavior in the whole population both at the investment stage and at the distribution stage. A norm of investment and a norm of division must therefore coevolve in the two games, supporting both the efficient investment profile and the egalitarian distribution of the surplus, respectively. The two games differ depending on the conditions needed for the two norms to coevolve. The games are proposed to explain the social norms used in modern hunter-gatherer societies.
\end{abstract}

Key Words: evolution; social norms; stochastically stable equilibrium; modern hunter-gatherer societies.

Codes. C78, D83, L14, Z13.

\footnotetext{
${ }^{1}$ Permanent Address: Ministry of Economy and Finance, via Solferino 11, 47121 Forli, Italy.

${ }^{\dagger}$ Corresponding Author; tel. +39 543374110; fax +39 0543374119; e-mail: giorgio.negroni@unibo.it
} 


\section{Introduction}

In this paper we study a two-stage game with two risk-neutral players in which a production phase precedes a division phase. In stage one both players have to simultaneously decide whether to invest or not; in stage two, after observing the gross surplus produced, they have to decide how to divide it. The game has a multiplicity of equilibria; players thus face a serious equilibrium selection problem which may nevertheless be solved if adequate social norms are in place (Binmore, 1998, 2007; Binmore and Shaked, 2010). Since in our model agents strategically interact in each stage of the game, the social norm of interest is twofold: it is a norm of cooperation, which dictates how to play the production stage, and a norm of distribution, which dictates how to divide the surplus produced. Examples of social norms of this kind can be found in the anthropological literature on cooperative food acquisition and cooperative hunting in modern hunter-gatherer societies. For instance the Ache of Paraguay developed a rule of thumb for hunted resources of the kind "cooperate frequently and share fully" (Hill, 2002); a similar conclusion, related to whale hunters in Lamelera, is drawn by Alvard and Nolin (2002). The observed compliance to these rules of thumb by several hunter-gatherers has led some social scientists to suggest that these norms must have been evolving over time, probably as a way to regulate large-game hunting (Bohem, 2004). Our goal is to explore whether and under what conditions social norms supporting both an efficient outcome and neat distributional rule can endogenously arise through an evolutionary process.

We assume that the investment is costly and, to simplify the analysis, that the cost is the same for both agents. The surplus depends on the investment profile. We further assume that when nobody invests no surplus is produced and both agents receive nothing. Thus, a bargaining stage only occurs when at least one agent has invested in the first stage.

Two different extensive games, corresponding to two different structures of political and social organization, are considered. In both extensive games we assume that when the two players have chosen to invest the bargaining stage follows the rule of the Nash Demand Game (NDG). When, on the other hand, an asymmetric investment profile is observed two simple alternatives are considered. Each of these specifies how the unique investing agent can reap the rewards of her own action.

In the first alternative we assume that the sociopolitical organization promotes full right of possession. As a consequence all the bargaining power is assigned to the player who has decided to invest; by allowing her to behave as a dictator, the bargaining stage then collapses into a Dictator Game (DG). In this framework if a sharing occurs it only depends on the free will of the unique investing agent. In the second alternative we assume that the sociopolitical organization recognizes a right to sharing by asking an agreement across agents as condition to gather the surplus. However the agreement has to emerge in a situation in which, due to the recognized right, the bargaining power is asymmetric. This suggests that bargaining can take the form of an Ultimatum Game 
(UG) in which the player who has chosen to invest proposes a distribution which is only realized if the opponent accepts it otherwise the surplus is lost due to (unspecified) conflict. By $\Gamma_{D G}$ (resp. $\Gamma_{U G}$ ), we denote the whole game in which an NDG occurs when a symmetric investment profile is observed, and a DG (resp. UG) occurs when an asymmetric investment profile is observed. Both games $\left(\Gamma_{D G}\right.$ and $\left.\Gamma_{U G}\right)$ have a multiplicity of equilibria.

The anthropological literature suggests that $\Gamma_{D G}$ can be appropriate in a society in which production is a collective venture and property rights are secured, as it seems to happen among the Mbuti pygmies (Ichikawa, 1983); on the other hand $\Gamma_{U G}$ can be suitable for a society in which production is an individual activity but in which full right of possession is not supported, as it seems to be the case for the !Kung (Woodburn, 1982).

In order to identify the evolved social norms we use the concept of stochastic stability and we apply the evolutionary framework for extensive games put forward by Noldeke and Samuelson (1993). We claim that a social norm has evolved when the stochastically stable set only supports an homogeneous behavior for at least one population. Our main result states that in both games (i.e. $\Gamma_{U G}$ and $\Gamma_{D G}$ ) when a social norm evolves then not only do we observe an homogeneous behavior along the whole path of play but the prescribed actions are uniform across populations. We can thus claim that in both games the norms coevolve; in this case the investment norm supports full cooperation in the production stage (meaning that both agents choose to invest) and the bargaining norm entails an egalitarian division of the realized surplus. The two games differ depending on the conditions needed for the two norms to coevolve. In particular we always observe a coevolution of norms in $\Gamma_{D G}$ whereas in $\Gamma_{U G}$ this only occurs when investments are complements and the cost of investment is not too high; when these conditions fail a great deal of outcomes are stochastically stable so that neither an investment norm nor a bargaining norm evolve.

Our main results are derived under the natural assumption that full cooperation in the production stage is efficient; this means that the net surplus observed when both agents choose to invest is greater than the net surplus when only one invests. Recently Avard (2004) advanced the hypothesis that norms of cooperation and norms of fair division were more likely to be observed in societies in which "the payoffs to cooperation are high". In order to verify Alvard's hypothesis, in the last Section we very briefly extend our analysis to the case in which full cooperation in the production stage is not efficient and we show that our results do not change.

The basic model is presented in Section 3. Section 4 describes evolutionary dynamics and gives some preliminary results. The main results are provided in Section 5, and further insights are discussed in Section 6. In the next Section 2 we briefly relate our model to the relevant literature. 


\section{Relation to literature}

Troger (2002), Ellingsen and Robles (2002) and Dawid and MacLeod (2001, 2008) investigate the consequences of evolutionary dynamics in scenarios characterized by investment specificity ${ }^{3}$. Broadly speaking this literature has shown that evolution (i.e. stochastic stability) may or may not support an efficient investment profile depending on whether only one (Troger, 2002; Ellingsen and Robles, 2002) or both parties (Dawid and MacLeod, 2008) make a relationspecific investment in the project, respectively. However any further comparisons between these models is hindered by the fact that the games structure is rather different.

In particular, Ellingsen and Robles (2002) and Troger (2002) consider the case in which total output is determined by the decision of one agent only and the bargaining proceeds according to the rule of NDG. They have shown that all the stochastically stable equilibria are efficient and that a neat distributive norm evolves virtually assigning all the surplus to the investor provided that a fine grid of possible investments is allowed. When the size of the pie is endogenous, the aforementioned papers have proved an important result, namely that separating the analysis of the bargaining stage from the prior investment stage is illegitimate in the context of the hold-up literature.

Dawid and MacLeod (2001) is the contribution closer to our model. They consider a two-stage game with two-sided relation-specific investment and show that the efficiency result proved by Ellingsen and Robles (2002) and Troger (2002) may not extend to this case. However in our opinion their way of shaping the distribution stage is rather critical. Indeed they assume that equal split occurs after a symmetric investment profile while a bargaining game, which follows the rule of the NDG, only occurs after an asymmetric investment profile. We argue that, though the equal split at symmetric investment profile may be an adequate hypothesis, it sweeps away the main issue namely the origin of this social norms when total surplus depends on previous investment decisions ${ }^{4}$. When instead an asymmetric investment profile is observed, the assumption that they haggle according to the rule of NDG is tantamount to assume a structure of individual preferences more biased towards free-riding demeanor; although this assumption can be adequate in some context we contend its general validity.

These assumptions, coupled with the considered evolutionary dynamics, make the whole model unsuitable for studying the evolution of a bargaining norm; this follows directly from the fact that, although in Dawid and MacLeod (2001) bargaining only occurs when asymmetric investment profiles are observed, no limit set supporting this asymmetric profile exists under their unperturbed dynamics. ${ }^{5}$ Dawid and MacLeod (2008) is a further extension in which

\footnotetext{
${ }^{3}$ We say that investment is completely relation-specific when it is only valuable to a particular trading partner. It is well known that investment specificity, making the investor vulnerable to ex-post exploitation, may give rise to the so-called hold-up problem.

${ }^{4}$ When the size of pie is exogenously given Young (1993) has shown that equal split emerges as bargaining norm.

${ }^{5}$ The evolutionary dynamics they use are an adaptation of Young (1993a) to extensive
} 
the outcome of the investment decision is stochastic. They show that (i) the bargaining norm affects the investment norms but the opposite is not true; (ii) the problem of under investment is stricter when investments are complements.

As we said, this literature is concerned with (one-sided or two-sided) relationspecific investment. By contrast our main interest is to study a generic setting in which people are not dependent on specific other people for access to basic requirements and in which individuals are not bound to fixed assets or fixed resources. This makes our model more apt for describing simple societies as modern hunter-gatherers (Woodburn, 1982), for instance. Notwithstanding, if some scholar want to apply our models to two-sided relation-specific investment then we suggest to consider $\Gamma_{U G}$ only. In fact, it is only in this game that the single agent who has invested is vulnerable to ex-post exploitation. ${ }^{6}$ It is worth noting that, although the formal conditions for a stochastically stable outcome to exist in $\Gamma_{U G}$ coincide with those required by Dawid and MacLeod (2001), the basic models and evolutionary dynamics are different. In particular, while in $\Gamma_{U G}$ these conditions support the coevolution of a norm of investment and a norm of distribution, they only uphold a norm of investment in Dawid and MacLeod (2001).

\section{The model}

In this Section we describe strategic environments; in the next, these will be embedded in an evolutionary framework. Two risk neutral players $(A$ and $B)$ are engaged in a two stage game. In stage one, both have to simultaneously decide whether to invest (action $H$ ) or not (action $L$ ); when a player chooses $H$ she incurs cost $c$. A surplus is produced and observed at the end of stage one; each player can then correctly estimate her opponent's investment. We denote the surplus arising when both choose $H$ by $V_{H}$; the surplus accruing when only one chooses $H$ by $V_{M}$; and lastly, when both choose $L$, by $V_{L}=0$. Obviously, $V_{H}>V_{M}>0$.

In stage two, they bargain over the available surplus. The bargaining rule depends on the investment profile. If both have chosen $H$, they are engaged in

form games. Although this extension is not problematic with one sided investment (as in Troger, 2002), it is a bit tricky with two sided relation-specific investment since it can imply that some agents can continue to believe that all the opponents make the same investment (i.e. all choose high or low investments) even when some bargaining outcomes (which in their model can only happen when high-low matches occur) are observed.

${ }^{6}$ Ellingsen and Robles (2002) also considered the case in which, in stage two, the distribution of the surplus is determined by an ultimatum game where the player who makes the proposal is the trading partner, i.e. the agent not responsible for the investment decision. They have shown that in this case the stochastic stability has little cutting power because many outcomes are stochastically stable. Our game $\Gamma_{U G}$ mainly differs from Ellingsen and Robles (2002) in two respects. First, the player who makes the proposal is the player who decided to invest in the first stage. Second, since both agents can decide to invest, both can be in a position to affect the distribution of the surplus generated by the other. Our result for $\Gamma_{U G}$ says that, under the appropriate conditions, a unique stochastically stable outcome exists. 
a Nash Demand Game (NDG). If they have chosen different investments, two alternatives are conceivable: an Ultimatum Game (UG) and a Dictator Game (DG). We denote $\Gamma_{U G}$ the whole extensive game in which a NDG occurs when both players have invested while a UG occurs when only one player has invested. Analogously we denote $\Gamma_{D G}$ the whole extensive game in which a DG occurs when only one agent has invested. ${ }^{7}$ Let $D\left(V_{j}\right)=\left\{\delta, 2 \delta, \ldots, V_{j}-\delta\right\}, j \in\{H, M\}$ denote the set of feasible claims.

Throughout the paper we consider a class of games in which the following Assumption holds:

Assumption 1 (a) $\frac{V_{H}}{2}$ and $c$ are divisible by $\delta$ and $c>\delta$;

(b) the efficient net surplus arises when both players choose $H$, i.e.

$$
c<\min \left(\frac{V_{H}}{2} ; V_{H}-V_{M}\right) ;
$$

(c) the maximum payoff attainable by playing $H$ when the opponent chooses $L$ is not negative, i.e.

$$
c \leq V_{M}-\delta .
$$

In NDG, both players simultaneously make $y$ and $x$ demands. If the demands are compatible, each receives what she claimed; otherwise they receive nothing. When both choose $H$, the payoffs are

$$
\pi_{A}=\left\{\begin{array}{ccc}
y-c & \text { if } & y+x \leq V_{H} \\
-c & \text { if } & y+x>V_{H}
\end{array}\right.
$$

and

$$
\pi_{B}=\left\{\begin{array}{ccc}
x-c & \text { if } & y+x \leq V_{H} \\
-c & \text { if } & y+x>V_{H} .
\end{array}\right.
$$

In UG the player who has chosen $H$ makes a proposal that the opponent can either accept or reject. Let's suppose $H L$ is observed and $A$ proposes the division $\left(y, V_{M}-y\right)$. If $B$ accepts, the payoffs are $y-c$ for $A$ and $V_{M}-y$ for $B$; otherwise $A$ gets $-c$ and $B$ nothing.

In DG, the player who has chosen $H$ decides a division her opponent cannot reject. Suppose $H L$ is observed and $A$ demands $y$. The payoffs are $y-c$ for $A$ and $V_{M}-y$ for $B$.

It is worth noting that, under Assumption 1, both $\Gamma_{U G}$ and $\Gamma_{D G}$ admit a subgame perfect equilibrium which supports investment profile $H H$. Nevertheless, both games admit a great deal of subgame perfect equilibria some of which are inefficient.

\footnotetext{
${ }^{7}$ We stress that this strategic environment is compatible with two kinds of overt behavior regards production. In one case, production calls for a collective activity, while in the other it arises from an individual activity. In both cases, the agents are aware that a distribution stage will follow. Both forms are present among modern hunter-gatherers.
} 


\section{Evolutionary dynamics}

In this Section we consider the evolutionary dynamics put forward by Noldeke and Samuelson (1993). To this end we postulate a finite population of size $N$ for each player, $A$ and $B$. In each period, every possible match between agents occurs meaning that each agent belonging to population $A$ interacts with each agent of population $B$, one at a time. An agent is described by a characteristic which consists of a detailed plan of action and a set of beliefs concerning the choice and the demands made by opponents in the first stage and in the second stage respectively. In $\Gamma_{U G}$, a plan of action for player $A$ must specify: (i) the type of investment; (ii) the demand when both players choose $H$ (the action at $H H$ ); (iii) the demand when $A$ chooses $H$ and $B$ chooses $L$ (the action at $H L$ ); (iv) whether to accept or reject any demands made by $B$, when in the first stage $B$ chooses $H$ and $A$ chooses $L$. The same applies for player $B$. In $\Gamma_{D G}$, a plan of action for player $A$ must specify: (i) the type of investment; (ii) the demand when both players choose $H$ (the action at $H H$ ); (iii) the division of the surplus when $A$ chooses $H$ and $B$ chooses $L$ (the action at $H L$ ). The same applies for player $B$. A state, $\theta$, is a profile of characteristics of the overall population. $z(\theta)$ is the probability distribution across terminal nodes, given the state of the population $\theta$. The set of possible states, $\Theta$, is finite.

At the end of every period each agent with probability $\lambda$ observes $z(\theta)$ and her characteristic may change. In particular the received information allows agents to correctly update their beliefs on opponent's choices at the observed information sets. Given their new beliefs, they also update their action profile by choosing a best reply ${ }^{8}$ at each information set. With probability $1-\lambda$ the single agent does not observe $z(\theta)$ and her characteristic does not change. This learning mechanism engenders an (unperturbed) Markov process $(\Theta, P)$ where $P$ is the transition matrix on $\Theta$.

By $\Omega$ we denote a generic limit set ${ }^{9}$ of the process; this is a minimal subset of states such that, when the process enters, it does not exit. By $\rho(\Omega)$ we denote the set of observable terminal nodes under $\Omega$. Lastly by $\Sigma$ we denote the union of the limit sets of the process .

Besides being updated, agents' beliefs and actions can also change by mutation. In every period, each agent has a probability $\epsilon$ of mutating. Mutations are independently distributed across agents. When mutating, agent changes her characteristic according to a probability distribution assigning positive probability on each possible characteristic.

A particular type of mutation occurs when the mutants change belief and/or action at some unreached information set under the current state $\theta$. In this case we assert that the state drifts.

\footnotetext{
${ }^{8}$ However if the learning agent has already played a best reply her action does not change. Moreover when the best reply contains more than one action, one of these can be randomly chosen according to a distribution with full support.

${ }^{9} \mathrm{~A}$ set $\Omega \subseteq \Theta$ is called a limit set of the process $(\Theta, P)$ if: (a) $\forall \theta \in \Omega$, $\operatorname{Prob}\left\{\theta_{t+1} \in \Omega \mid \theta_{t}=\theta\right\}=1$; (b) $\forall\left(\theta, \theta^{\prime}\right) \in \Omega^{2}, \exists s>0$ s.t. $\operatorname{Prob}\left\{\theta_{t+s}=\theta^{\prime} \mid \theta_{t}=\theta\right\}>0$.
} 
Mutations generate a new (perturbed) Markov process $(\Theta, P(\epsilon))$, which is ergodic. It is well known that, for any fixed $\epsilon>0$, the perturbed process has a unique invariant distribution $\mu_{\epsilon}$. Let $\mu_{*}=\lim _{\epsilon \rightarrow 0} \mu_{\epsilon}$ denote the limit distribution. A state $\theta$ is stochastically stable if $\mu_{*}(\theta)>0$. We denote the set of stochastically stable states by $\Sigma_{S}$; this is the set of states which has a positive probability in limit distribution. Noldeke and Samuelson (1993) proved that the stochastically stable set is contained in the union of the limit sets of the unperturbed process. In order to detect the stochastically stable set we first have to characterize the limit sets of our model; this is the aim of the following two Propositions.

Proposition 2 In $\Gamma_{U G}$ all the limit sets have one of the following structures: (a) they contain one state only, and this is a self-confirming equilibrium of the game; (b) they contain more than one state and all investment profiles are observed. Moreover, only one outcome is realized for each investment profile in which the claims exhaust the surplus. ${ }^{10}$

Proof. See the Appendix

Proposition 3 In $\Gamma_{D G}$ all the limit sets contain one state only, and this is a self-confirming equilibrium. Moreover, at least one agent chooses to invest at every equilibrium.

Proof. See the Appendix

From now on when we speak of equilibrium we refer to self-confirming equilibrium. $^{11}$

Propositions 2 and 3 state that the considered evolutionary dynamic gives rise to a large multiplicity of limit sets. However, this dynamic admits limit sets in which both investment and bargaining behavior is uniform in each population. It is thus likely that homogeneous behavior in one or both populations could be molded by evolution. When this happens, we say that a norm has evolved. Accordingly, an investment norm has evolved if all agents belonging to the same population make the same investment and the investment behavior is correctly anticipated . Analogously, a bargaining norm has evolved if a pair of demands $(y, x)$ exists at some reached information set which exhausts the gross surplus and the bargaining behavior is correctly anticipated. When the

\footnotetext{
${ }^{10}$ Careful reading of the Proposition proof shows that the claims must satisfy a well-defined set of constraints.

${ }^{11}$ According to Noldeke and Samuelson (1993) a state is a self-confirming equilibrium if each agent's strategy is a best response to that agent's conjecture and if each agent's conjecture about opponent's strategies matches the opponent's choices at information sets that are reached in the play of some matches.
} 
set of stochastically stable states contains only equilibria supporting the same outcome we speak of a stochastically stable outcome rather than a stochastically stable set.

Despite the fact that Propositions 2 and 3 do not help to pin down which behavior is more likely to become the conventional one, in the next Section we shall show that the stochastically stable set can support only one outcome in both games. Our remarkable result stems from direct application of Noldeke and Samuelson (1993) and Ellison (2000). In particular, Proposition 1 of Noldeke and Samuelson (1993) states that if $\Sigma_{S}$ is a strictly subset of $\Sigma$ (i.e. $\Sigma_{S} \subset \Sigma$ ) then a $\Omega \notin \Sigma_{S}$ can not be reached from $\Sigma_{S}$ by a sequence of single-mutation transitions. Hence, our first task (Lemma 4 and 5 below) is to detect the smallest $\widehat{\Sigma} \subset \Sigma$ such that a sequence of a single-mutation transitions is enough to escape from any $\Omega \in \Sigma \backslash \widehat{\Sigma}$ and reach $\widehat{\Sigma}$. In this case when $\Sigma_{S} \subset \Sigma$ we know that $\Sigma_{S} \subset \widehat{\Sigma}$. From now on, by slightly abusing notation, $\left(H H, y_{H H}, x_{H H}\right)$ denotes a terminal node in which both agents have chosen $H$, agent $A$ makes a demand $y_{H H}$ and agent $B$ makes a demand $x_{H H}$. This applies for the other terminal nodes, too.

The next Lemma states our first preliminary result, which holds true for both the extensive games considered

Lemma 4 Consider a limit set $\Omega$ such that $\rho(\Omega)$ is not a singleton. An equilibrium supporting one outcome only can be reached from $\Omega$ by a sequence of single-mutation transitions.

Proof. See the Appendix.

Lemma 4 enables us to limit our attention to the equilibria supporting one outcome only. According to the investment profile observed, we can partition this set of equilibria into four subsets. We note these subsets respectively as $\Sigma_{H}, \Sigma_{L}, \Sigma_{H L}$ and $\Sigma_{L H}$. Of course, $\Sigma_{H}$ includes all the equilibria supporting the outcome $\left\{H H, V_{H}-x_{H H}, x_{H H}\right\}$ where $x_{H H} \in D_{\delta}\left(V_{H}\right)$. The same applies for the other subsets. The following Lemma 5 highlights that the process can move from any equilibrium belonging to the set $\left(\Sigma_{L} \cup \Sigma_{H L} \cup \Sigma_{L H}\right)$ to a new equilibrium $\theta \in \Sigma_{H}$ through a sequence of single-mutation transitions.

Lemma 5 Consider an equilibrium $\theta$; then:

(a) if $\theta \in \Sigma_{L}$ an equilibrium $\theta^{\prime} \in \Sigma_{H}$ can be reached from $\theta$ by a sequence of single-mutation transitions provided that $c+\delta<x_{H H}<V_{H}-c-\delta$;

(b) if $\theta \in \Sigma_{H L}$ (resp. $\Sigma_{L H}$ ) an equilibrium $\theta^{\prime} \in \Sigma_{H}$ can be reached from $\theta$ by a sequence of of single-mutation transitions provided that $c+\delta<x_{H H}<$ $V_{H}-c-\delta$.

Proof. See the Appendix. 
It is worth stressing that since subset $\Sigma_{L}$ is empty in $\Gamma_{D G}$, then only point (b) of the previous Lemma is relevant to this game.

Lastly Lemma 6 below asserts that in $\Gamma_{U G}$ a sequence of single-mutation transitions is enough to shift the process from any equilibrium $\theta \in \Sigma_{L}$ to a new equilibrium $\theta^{\prime}$ in which only one agent has invested.

Lemma 6 Consider $\Gamma_{U G}$ and an equilibrium $\theta \in \Sigma_{L}$ then an equilibrium $\theta^{\prime} \in$ $\Sigma_{L H} \cup \Sigma_{H L}$ can be reached from $\theta$ by a sequence of single-mutation transitions provided that at $\theta^{\prime}$ the agent who has chosen $H$ is better off;

Proof. See the Appendix.

All the results so far obtained only require that $c \leq \min \left(V_{M}-\delta, \frac{V_{H}}{2}-\delta\right)$. From Lemma 4 we know that, when a limit set underpins a multiplicity of outcomes, then we can reach an equilibrium sustaining only one outcome by a sequence of single mutations. Lemma 5 tells us that if the single equilibrium outcome does not support the efficient investment profile, then the process can reach a single equilibrium belonging to $\Sigma_{H}$ by a sequence of single mutations. Hence, when $\Sigma_{S} \subset \Sigma$, both these Lemma suggest we focus on subset $\Sigma_{H}$.

\section{Main results}

The conclusion of the previous Section suggests we limit our concern to the set of equilibria $\Sigma_{H}$ for both games. In this Section we show that when a unique stochastically stable outcome exists it always supports the efficient investment profile and the egalitarian distribution rule.

In this Section, we introduce a further technical assumption.

Assumption 7 The population is sufficiently large, i.e.

$$
\frac{V_{H}}{N}<\delta
$$

First and foremost, we provide a characterization of the stochastically stable set for $\Gamma_{U G}$; we then briefly consider $\Gamma_{D G}$. A number of further definitions are needed. By $x_{B}^{*}$ (resp. $V_{H}-x_{A}^{*}$ ) we denote the share going to player $B$ (resp. $A$ ), such that she receives a payoff equal to $\left(V_{M}-\delta\right)$ when both agents have invested:

$$
\begin{aligned}
& x_{B}^{*}=V_{M}-\delta+c \\
& x_{A}^{*}=V_{H}+\delta-c-V_{M} .
\end{aligned}
$$


Since $c$ is divisible by $\delta$ and $c>\delta$, then $x_{B}^{*}, x_{A}^{*} \in D_{\delta}\left(V_{H}\right)$. Let ${ }^{12}$

$$
\begin{aligned}
& \widehat{x}_{A}=\max \left\{x \in D_{\delta}\left(V_{H}\right) \mid\left(V_{H}-x\right) \frac{N-1}{N}-c \geq V_{M}-\delta\right\} \\
& \widehat{x}_{B}=\min \left\{x \in D_{\delta}\left(V_{H}\right) \mid x \frac{N-1}{N}-c \geq V_{M}-\delta\right\} .
\end{aligned}
$$

Under assumption (3) it follows that $\widehat{x}_{A}=x_{A}^{*}-\delta$ and $\widehat{x}_{B}=x_{B}^{*}+\delta$. Therefore $\widehat{x}_{B} \leq \widehat{x}_{A}$ if

$$
V_{M} \leq \frac{V_{H}}{2}-c .
$$

When this condition holds, then we can define the following set:

$$
\Sigma_{I H}=\left\{\theta \in \Sigma_{H} \mid x \in\left[\widehat{x}_{B}, \widehat{x}_{A}\right]\right\} .
$$

It is worth noticing that $V_{H}-\widehat{x}_{A}=\widehat{x}_{B}$, meaning that in $\Sigma_{I H}$ the lowest share of surplus is the same for both agents. Few computations show that $\frac{V_{H}}{2} \in \Sigma_{I H}$.

By definition when $\theta \in \Sigma_{I H}$ each agent receives an equilibrium payoff not smaller than the maximum payoff attainable when she deviates by playing $L$. Any equilibrium in $\Sigma_{I H}$ thus dominates all the equilibria supporting other investment profiles. Hence even if at an equilibrium $\theta \in \Sigma_{I H}$ the belief on the outcome in high-low matches drifts, allowing some agents to expect to get almost the whole surplus if they do not invest, this drift does not push the process away from the basin of attraction of $\theta$.

Let $\Sigma_{C H}=\Sigma_{H} \backslash \Sigma_{I H}$ be the subset of $\Sigma_{H}$ such that $x \notin\left[\widehat{x}_{B}, \widehat{x}_{A}\right]$. The next Lemma 8 shows that both $\Sigma_{I H}$ and $\Sigma_{C H}$ have some desirable features.

Lemma 8 Consider $\Gamma_{U G}$. Then:

a) under condition (6) more than one mutation is needed to escape from $\Sigma_{I H}$

(b) one mutation is enough to escape from $\Sigma_{C H}$ and to reach $\Sigma_{L}$;

(c) under condition (6), $\Sigma_{I H}$ can be reached from $\theta \in \Sigma_{C H}$ by a sequence of single mutations.

Proof. See the Appendix.

The next Proposition states our main result for $\Gamma_{U G}$. In order to derive this Proposition, we make use of both the sufficient condition developed by Ellison (2000) and some of the results for the NDG proved by Young (1993).

Proposition 9 Consider $\Gamma_{U G}$. If $\delta$ is sufficiently small and $V_{M} \leq \frac{V_{H}}{2}-c$ then a stochastically stable outcome exists. In this case all agents choose to invest and the surplus is equally split.

\footnotetext{
${ }^{12} \widehat{x}_{A}$ is the largest demand agent $B$ can make at $H H$ such that $A$ does not have any incentive to change action by playing $L$ when she knows that: (i) $N-1$ agents $B$ play $H$ and claim $\widehat{x}_{A}$; (ii) one agent $B$ makes a larger demand. Analogously for $\widehat{x}_{B}$.
} 
Proof. See the Appendix

Proposition 9 holds provided that the set $\Sigma_{I H}$ includes at least one equilibrium, condition satisfied when (6) holds. According to Dawid and MacLeod (2008), the investments are complements if the marginal effect of action $H$ when the opponent always plays $H$ is greater than the marginal effect of action $H$ when the opponent plays $L$. Since in our model $V_{L}=0$, this condition is met when $V_{M}<\frac{1}{2} V_{H}$. This in turn implies that only the efficient investment profile $H H$ supports a Pareto dominant equilibrium when $\delta$ is sufficiently small. Sure enough when $\Sigma_{I H}$ is not empty investments are complements in the sense of Dawid and MacLeod (2001), but investments can be complements in the sense of Dawid and MacLeod also when $\Sigma_{I H}$ is not well defined.

We now turn to the case in which a Dictator Game (instead of an Ultimatum Game) is played when an asymmetric investment profile is reached. Lemma 10 below points out a desirable propriety for some equilibria belonging to $\Sigma_{H}$.

Lemma 10 Consider $\Gamma_{D G}$ and let $\theta$ be an equilibrium belonging to $\Sigma_{H}$. When, at $\theta$, the distribution rule satisfies the following condition

$$
(\delta+c) \frac{N}{N-1}<x<V_{H}-(\delta+c) \frac{N}{N-1}
$$

then more than one mutation is needed in order to reach an equilibrium supporting a different set of outcomes.

Proof. see the Appendix

Lemma 10 suggests that also for $\Gamma_{D G}$ we can identify a subset of $\Sigma_{H}$ with the property that more than one mutation is required to escape from it. As before we denote this subset by $\Sigma_{I H}^{D}$. The same argument used above shows that $\Sigma_{I H}^{D}$ is well defined when ${ }^{13}$

$$
\delta<\frac{V_{H}-2 c}{4} .
$$

Consequently, when $\delta$ is sufficiently small and the rules of the game give all the bargaining power to the only agent who has played $H$, then no further assumptions on the cost of investment are needed in order to argue that the most efficient investment norm evolves in the long run. The following Proposition summarizes our finding concerning $\Gamma_{D G}$.

Proposition 11 Consider $\Gamma_{D G}$. If $\delta$ is sufficiently small and $c \leq V_{M}-\delta$ then a stochastically stable outcome always exists and it supports full investment and the egalitarian distributional rule.

\footnotetext{
${ }^{13}$ See the Appendix for the details.
} 


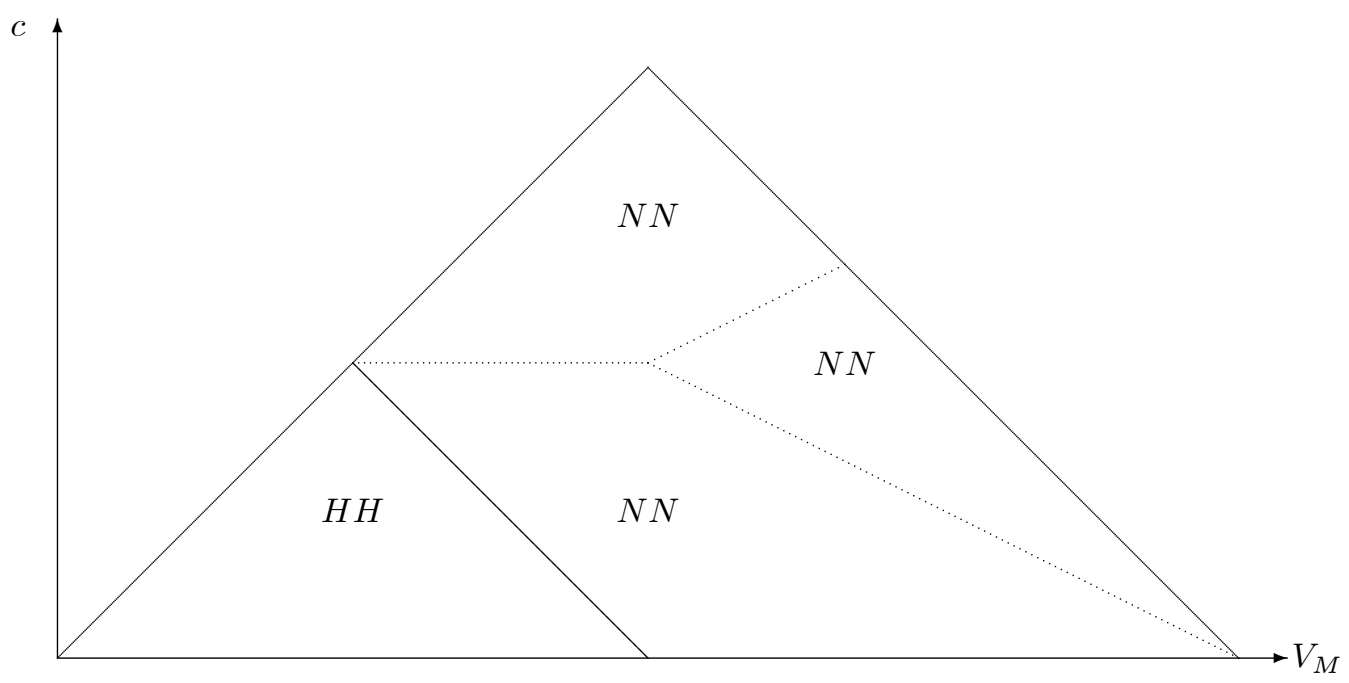

Figure 1: game $\Gamma_{U G}$. Parameter regions supporting the unique stochastically stable outcome $(\mathrm{HH})$ compared with the region $(\mathrm{NN})$ where no norm evolves since several stochastically stable outcomes can exist

Proof. See the Appendix

Proposition 11 states an intuitive result. Indeed in $\Gamma_{D G}$ the unique stochastically stable outcome is always $\left(H H, \frac{V_{H}}{2}, \frac{V_{H}}{2}\right)$ even when condition (6) does not hold. This implies that for whatever beliefs off the path (i.e. at $H L$ and/or $L H)$ the process still sustains the outcome $\left(H H, \frac{V_{H}}{2}, \frac{V_{H}}{2}\right)$. In particular, suppose that in period $t$ the process is at an efficient equilibrium $\theta$ in which the surplus is equally split but condition (6) does not hold. Let one $B$ agent expect to get almost the whole surplus if she does not invest. In period $t+1$ she will not invest. However, since at $H L$ all $A$ agents behave like a dictator, they will get almost the whole surplus. Hence in period $t+2$, by letting all $B$ agents revise, they all will choose to invest. Therefore the process does not leave the basin of attraction of $\theta$.

\section{Discussion}

In this Section, we provide further insights into our results. First consider $\Gamma_{U G}$. Borrowing from anthropological literature, we have suggested that this game is more apt for describing the evolution of investment and distribution norms when full rights of possession are not socially supported. Proposition 9 describes the scenarios we can expect to observe over the long run in a society of this kind ${ }^{14}$.

\footnotetext{
${ }^{14}$ Our results can be compared with Dawid and MacLeod (2001). If we put the assumptions $V_{L}=0$ and $V_{M}-c>0$ into their model, then the formal conditions for a single stochastically stable outcome stated in their Proposition 7 are in line with those stated in our Proposition
} 
First, either a norm of investment and a norm of bargaining coevolve, or no norm evolves at all. Second, when norms coevolve, they support efficient investment and egalitarian distribution. However, we can expect norms to coevolve when investments are complements (i.e. $V_{M}<\frac{1}{2} V_{H}$ ) and the investment cost is sufficiently low $\left(c<\frac{V_{H}}{2}-V_{M}\right)$. These conditions are satisfied in region $H H$ of Figure 1, which is drawn under the assumption that $\delta$ is negligible. Instead, when investments are complements but the investment cost is higher than $\frac{V_{H}}{2}-$ $V_{M}$, or when investments are not complements (i.e. $\frac{V_{H}}{2}<V_{M} \leq V_{H}$ ), we do not observe any norm. This happens in region $N N$ of Figure 1. In this region, since Lemmas 5, 6 and 8 (point b) are still true, we are sure that any equilibrium supporting a unique outcome belongs to the support of the limit distribution $\mu_{*}$. This means that all investment profiles and any distributional rule can be observed in the long run.

To see this consider region $N N$ and suppose ${ }^{15}$ that the only outcome observed is $\left(H H, \frac{V_{H}}{2}, \frac{V_{H}}{2}\right)$. Since no high-low matches are observed, the beliefs on bargaining outcomes in high-low matches can drift. Because of said drift, all agents $B$ (resp. $A$ ) might, for instance, deem that $A$ (resp. $B$ ) will only accept a distribution granting her almost the whole pie at $L H$ (resp. $H L$ ) profile even if all $B$ (resp. $A$ ) are ready to accept any proposal at $H L$ (resp. $L H)$. Sure enough the drift does not shift the process from the equilibrium outcome $\left(H H, \frac{V_{H}}{2}, \frac{V_{H}}{2}\right)$. Suppose now that one agent $B$ deems to capture a share $V_{M}-\delta$ at $H L$. According to this new belief she will play $L$ in the next period. Therefore the initial equilibrium $\left(H H, \frac{V_{H}}{2}, \frac{V_{H}}{2}\right)$ can be overturned when both populations update, once at the time, so that the process enters the basin of attraction of $(L L, 0,0)$ eventually. However this cannot be a social norm since a single mutation is enough to move the system into the basin of attraction of another equilibrium supporting a single outcome with a different investment profile (see Lemma 5, point (a), and Lemma 6). And so on and so forth.

We have also seen that $\Gamma_{D G}$ is more appropriate for describing the evolution of investment and distribution norms when full rights of possession are socially protected. In this context, Proposition 11 ensures that norms always coevolve. This result is illustrated in Figure 2, in which region $N N$ of the previous Figure 1 disappears and is replaced by region $H H$. Therefore, region $H H$ now coincides with the whole parameter space compatible with our main assumptions. Indeed, since in this case the only agent who has invested behaves as a dictator, any drift of beliefs does not upset the outcome $\left(H H, \frac{V_{H}}{2}, \frac{V_{H}}{2}\right)$ even when condition

9. However their Proposition 7 is only concerned with the evolution of investment norms instead of the coevolution of investment and bargaining norms. As we said, this stems from deep differences between the two models and the evolutionary dynamics considered. In the preliminary version of the present paper we have also studied the model in which the surplus is equally split when both agents invest, as in Dawid and MacLeod (2001), but an Ultimatum Game occurs when only one agent has invested. In this case Proposition 9 continues to be true. Lastly Proposition 9 continues to hold even when in the UG the agent who makes a proposal is not the agent who has chosen to invest, as in Ellingsen and Robles (2002).

${ }^{15}$ We remind that in region $N N$ condition (6) does not hold. 


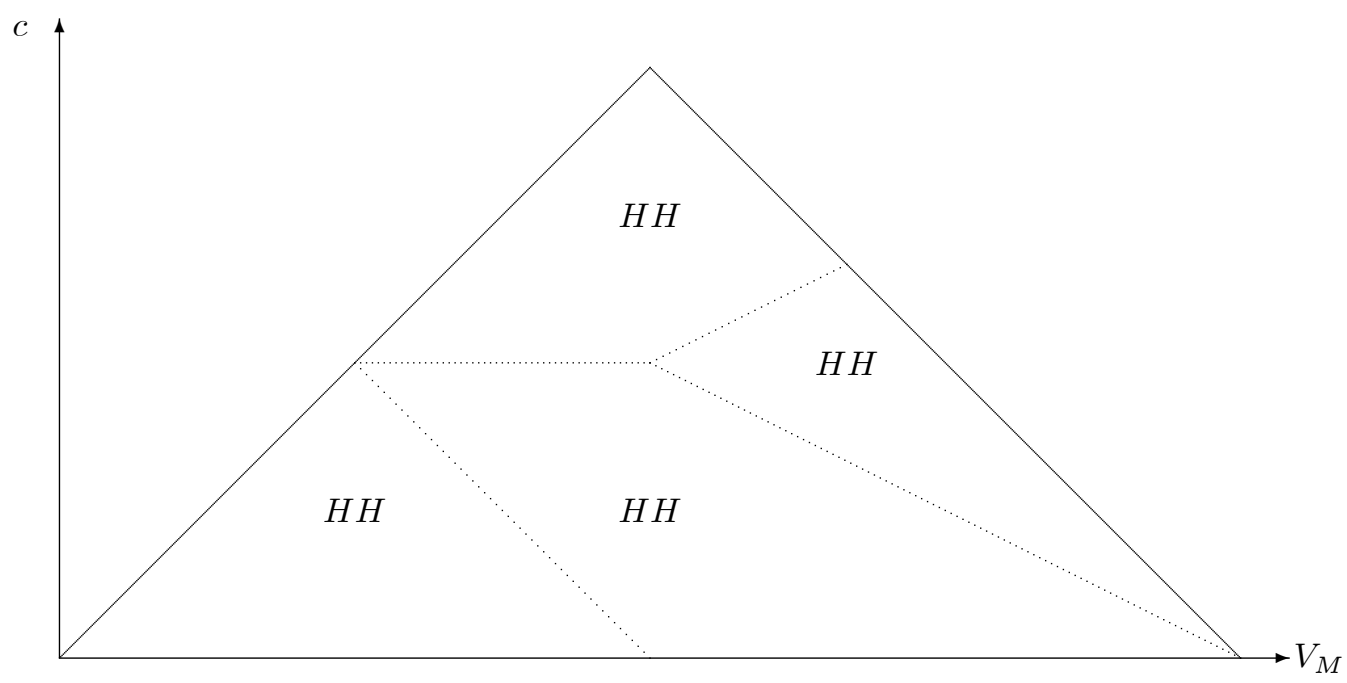

Figure 2: game $\Gamma_{D G}$. Parameter region supporting the only stochastically stable outcome $(\mathrm{HH})$.

(6) is not satisfied. Confidence in the dictator's unselfish behavior is thus always shortsighted.

In both games, when social norms emerge, they support the efficient investment profile and the egalitarian distribution of the surplus. The fact that the distributional norm is egalitarian is not a surprise since our model is symmetric. In turn this symmetry depends on the assumptions that investment costs are identical and that the surplus accrued when only one agent invests does not depend on the identity of the investing agent. One may then reasonably question whether an egalitarian norm could still be expected to emerge when some form of heterogeneity is allowed (Baker and Swope, 2005). This is an important point since the literature on modern hunters-gatherers suggests that, in some cases, these societies endorse an egalitarian rule of distribution even when differences in hunting-gathering abilities are observed (Kaplan and Hill, 1985; Woodburn, 1982) while in some other cases they do not (Hawkes, 1992). We have left this to further research.

Our results are derived under the natural assumption that full cooperation in the production stage is efficient. We now briefly provide some insights on its role; this allows us to evaluate a conjecture recently advanced by Alvard (2004). In discussing the results of some experiments in fifteen small scale societies ${ }^{16}$, Alvard (2004) suggested that norms of cooperation and norms of fair division were likely to be observed in societies in which "the payoffs to cooperation are

\footnotetext{
${ }^{16}$ See Heinrich et al. (2004). An assessment of these experiments can be found in Chibnik (2005) and in Hagen and Hammerstein (2006).
} 

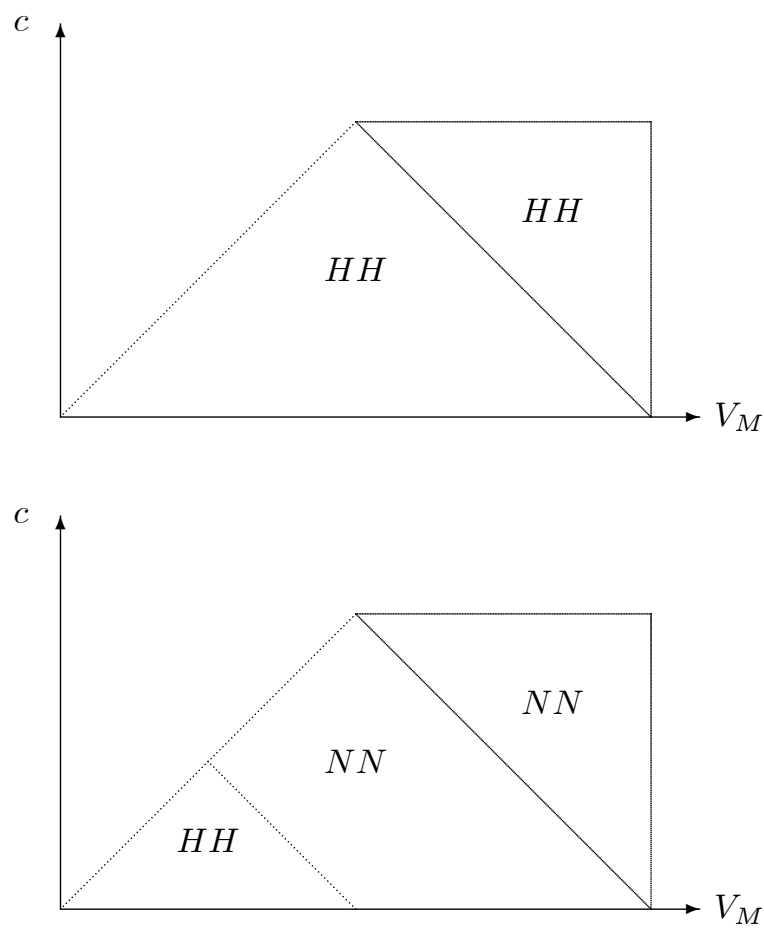

Figure 3: Top: game $\Gamma_{D G}$; bottom: game $\Gamma_{U G}$. In both Figures, the triangle on the right hand side denotes the parameter region for which investment by both agents is inefficient. No norms are observed in $\Gamma_{U G}$ while norms co-evolve in $\Gamma_{D G}$

high". Hence by relaxing our efficiency assumption and by comparing the results we can test the cutting power of Alvard's hypothesis in our settings. In order to do this we keep all the assumptions so far made with the exception of point (b) of Assumption 1 which is replaced by $V_{M}-c>V_{H}-2 c>0$. Under this new scenario all our intermediate results as well as point (b) of Lemma 8, Lemma 10 and Proposition 11 are still valid. However condition (6) can no longer be satisfied; as a consequence points (a) and (c) of Lemma 8 and Proposition 9 are not true. Therefore our results do not change. In $\Gamma_{D G}$ the same social norms coevolve with and without the efficiency hypothesis. In $\Gamma_{U G}$ no norm evolves in the added region due to the failure of condition (6); consequently region $N N$ enlarges. Both cases are illustrated in Figure 3 where the new parameter configuration is represented by the triangle added on the right hand side of each panel.

Lastly, we speculate on the role played by bargaining in the evolution of social norms in $\Gamma_{U G}$ and in $\Gamma_{D G}$ under the efficiency assumption. In order to do this, we apply the evolutionary framework to a simpler model in which a distributional norm already exists and agents only have to coordinate their 
investment decisions. To this end we consider the one shot game suggested by Hawkes (1992, payoff matrix 8). Let $\Gamma_{C}$ denote this model. The resulting game is represented by the normal form (9) in which we assume ${ }^{17}$ (i) that for whatever investment profile the pie is equally split ${ }^{18}$; (ii) that the net surplus arising when both agents invest is the greatest one, and (iii) that the net surplus accruing when only one invests is not negative. The latter two conditions are satisfied when $c<\min \left(\frac{1}{2} V_{H}, V_{H}-V_{M}, V_{M}\right)$. In this strategic framework, when there is more than one pure Nash equilibrium, the stochastically stable one coincides with the risk dominant equilibrium (Young, 1993a).

From an anthropological perspective, this game is suitable to describe a situation arising when the pie to be distributed has to be produced in advance but, since property rights cannot be enforced ${ }^{19}$, it must always be split equally.

$$
\begin{array}{c|cc}
\multicolumn{1}{c}{} & H & L \\
\cline { 2 - 3 } H & \frac{V_{H}}{2}-c, \frac{V_{H}}{2}-c & \frac{V_{M}}{2}-c, \frac{V_{M}}{2} \\
L & \frac{V_{M}}{2}, \frac{V_{M}}{2}-c & 0,0
\end{array}
$$

Few computations show that the game has either three Nash equilibria (two in pure strategies and one in mixed strategies) or only one pure symmetric Nash equilibrium. When the game admits a unique Nash equilibrium, this is investment profile $H H$ if $c<\min \left(\frac{V_{M}}{2}, \frac{V_{H}-V_{M}}{2}\right)$ and investment profile $L L$ if $c>\max \left(\frac{V_{M}}{2}, \frac{V_{H}-V_{M}}{2}\right)$. When the game admits two pure Nash equilibria, these are $H H$ and $L L$ if $\frac{V_{M}}{2}<c<\frac{V_{H}-V_{M}}{2}$ and $H L$ and $L H$ if $\frac{V_{H}-V_{M}}{2}<c<\frac{V_{M}}{2}$.

When the game has two pure symmetric equilibria, the stochastic stability selects one of them. In particular, it selects investment profile $H H$ if $\frac{V_{M}}{2}<c<$ $\min \left(\frac{V_{H}-V_{M}}{2}, \frac{V_{H}}{4}\right)$, while it selects investment profile $L L$ if $\max \left(\frac{V_{M}}{2}, \frac{V_{H}}{4}\right)<c<$ $\frac{V_{H}-V_{M}}{2}$. When instead the game has two pure asymmetric equilibria, $L H$ and $H L$, both are stochastically stable.

These results are illustrated in Figure 4, where $H H$ (resp. $L L$ ) denotes the region in which investment profile $H H$ (resp. $L L$ ) is the only stochastically stable equilibrium and where $N N$ denotes the region in which no investment norms emerge since both equilibria, $L H$ and $H L$, are stochastically stable. In this last game, a coordination failure arises when investment profile $L L$ is the only stochastically stable equilibrium. Direct comparison of Figures $1\left(\Gamma_{U G}\right)$ and 3 allows us to argue that the main consequences of modeling a bargaining

\footnotetext{
${ }^{17}$ This game tallies with Hawkes's game under the assumptions $V=V_{H}$ and $s V=V_{M}$.

${ }^{18}$ This means that the distributional rule in $\Gamma_{C}$ coincides with the unique distributional norm which can evolve in $\Gamma_{U G}$.

${ }^{19}$ According to the anthropologist, this situation is compatible with societies admitting the so-called tolerated theft (Hawkes, 1992). This means that sharing also occurs when the pie is only provided by one agent, since excluding outsiders is too costly. However, Bell (1995) argued that tolerated theft presumes that society ensures the hunter the full right to his or her catch, a condition that may not be granted.
} 


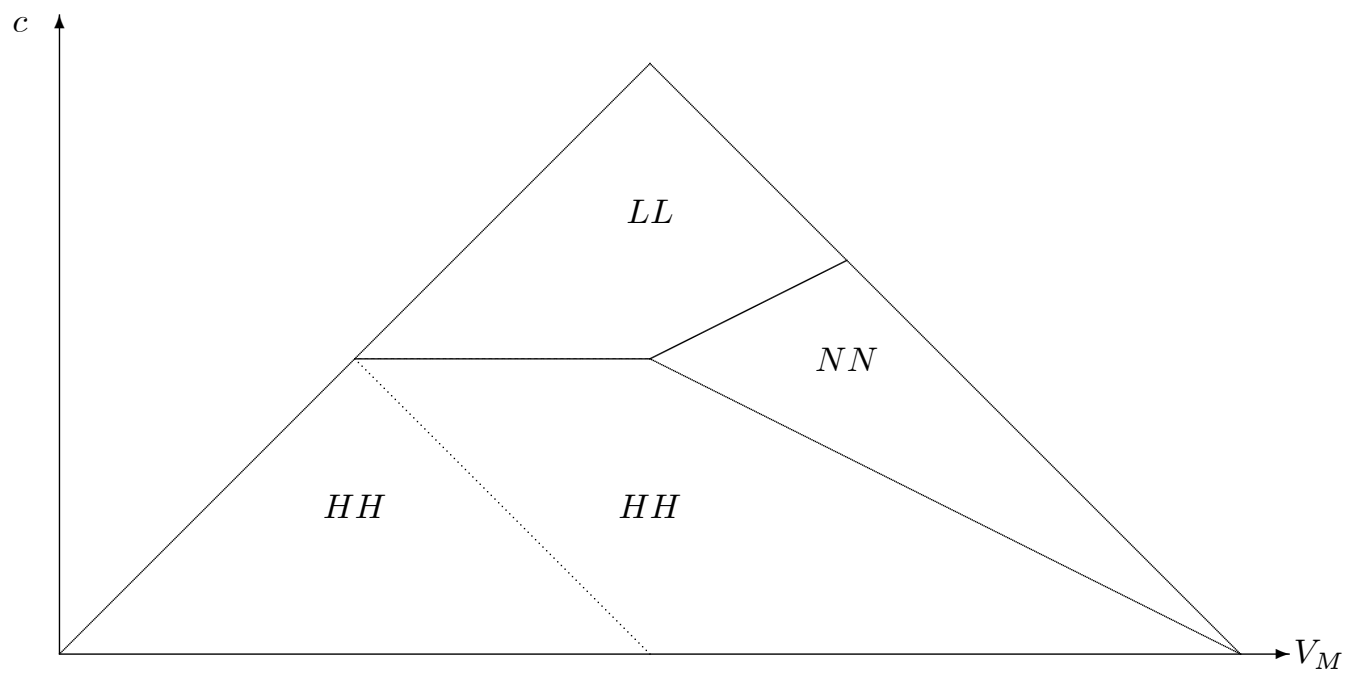

Figure 4: game $\Gamma_{C}$. The parameter regions where efficient investment $(\mathrm{HH})$ and no investment (LL) are stochastically stable compared with the region (NN) where no investment norm evolves.

stage as a game is that the region supporting the worst outcome $(L L)$ disappears while the region supporting the best outcome $(H H)$ shrinks. Hence, the region in which no investment and bargaining norms evolve is enlarged. This highlights the fact that, contrary to what happens in $\Gamma_{C}$ in which an egalitarian distributional norm is already established, when a distribution norm is allowed to evolve, its evolutionary process affects the evolution of the investment norm (Hackett, 1993, 1994).

When we do the same exercise for $\Gamma_{D G}$, we obtain a new game $\Gamma_{C}^{\prime}$ which differs from $\Gamma_{C}$. This is because at the asymmetric investment profiles, the whole surplus goes to the investing agent only. Under the structural assumptions of the paper, it turns out that $H$ is now a dominant strategy and $H H$ is the only Nash equilibrium. ${ }^{20}$ Since $\Gamma_{C}$ and $\Gamma_{C}^{\prime}$ essentially differ in the degree of property protection assured, we conclude that modeling a bargaining stage is crucial for understanding the evolution of both investment and distribution norms in societies in which full rights of possession are not socially supported. In societies in which full rights of possession are socially protected, this only explains which bargaining norm is brought to bear.

\section{Acknowledgments}

This paper was written while Lidia Bagnoli was research fellow of the Department of Economics, University of Bologna; financial support from DSE gratefully acknowledged. We would like to thank Ken Binmore and Kim Hill

\footnotetext{
${ }^{20}$ We remark that in $\Gamma_{C}^{\prime}$ any distributional norms granting a positive payoff when both invest does not hamper the evolution of the investment norm.
} 
for comments on an earlier version of this paper. The paper has been presented at a seminar held at the University of Bologna, at the annual meeting of the Italian Economists Society (Matera) and at the ASSET 2012 conference (Lymassol). We thank all participants for their comments; we are the only responsible for errors and imprecisions. 


\section{Appendix}

First of all we introduce some useful notations. Let us consider a state $\theta$ and suppose that all agents observe $z(\theta)$. In $\Gamma_{U G}$ action $L$ is not preferred to action $H$ for agents $i \in A$ if

$$
p_{B}(\theta)\left(\widetilde{y}_{H H}^{i}(\theta)-\widetilde{y}_{L H}^{i}(\theta)-c\right)+\left(1-p_{B}(\theta)\right)\left(\widetilde{y}_{H L}^{i}(\theta)-c\right) \geq 0 ;
$$

analogously, action $L$ is not preferred to action $H$ for agents $i \in B$ if

$$
p_{A}(\theta)\left(\widetilde{x}_{H H}^{i}(\theta)-\widetilde{x}_{H L}^{i}(\theta)-c\right)+\left(1-p_{A}(\theta)\right)\left(\widetilde{x}_{L H}^{i}(\theta)-c\right) \geq 0 .
$$

Here, by $p_{A}(\theta)$ (resp. $\left.p_{B}(\theta)\right)$ we denote the frequency of agent $A$ (resp. $B$ ) who played $H$ in $\theta$, and by $\widetilde{y}_{H H}^{i}(\theta)$ (resp. $\widetilde{x}_{H H}^{i}(\theta)$ ) the expected payoffs of agent $i \in A$ (resp. $i \in B$ ) at the information set $H H$, given $z(\theta)$. This applies for the other information sets, too. Similar conditions hold for $\Gamma_{D G}$.

The following preliminary results are needed in order to prove Propositions 2 and 3. Lemma 12 and Lemma 14 are needed for both games $\left(\Gamma_{U G}\right.$ and $\left.\Gamma_{D G}\right)$; Lemma 13 is needed for $\Gamma_{U G}$ only.

Lemma 12 Let $x_{H H, 1}<x_{H H, 2}<\ldots<x_{H H, k}$ be the demands made by $B$ at $\mathrm{HH}$ for some state $\theta$. Then the set of best behavioral demands following $\mathrm{HH}$ for agents $A$ is a subset of $\left\{V_{H}-x_{H H, l}\right\}_{l=1}^{k}$.

Proof. See Lemma A.1 in Ellingsen and Robles (2002).

Lemma 13 Consider $\Gamma_{U G}$ and let $\Omega$ be a limit set of $(\Theta, P)$. If $\left(H L, y_{H L}, x_{H L}\right) \in$ $\rho(\Omega)$ (resp. $\left.\left(L H, y_{L H}, x_{L H}\right) \in \rho(\Omega)\right)$ then:

(i) $x_{H L}=V_{M}-y_{H L}\left(\right.$ resp. $\left.y_{L H}=V_{M}-x_{L H}\right)$;

(ii) $\left(H L, y_{H L}, x_{H L}\right)$ (resp. $\left.\left(L H, y_{L H}, x_{L H}\right)\right)$ is the only outcome which supports investment profile $H L$ (resp. LH) in $\rho(\Omega)$.

Proof. We only consider profile $H L$. The same holds true for $L H$.

Point (i). Let $\theta$ be a state such that: (a) $\theta \in \Omega$; (b) $\left(H L, y_{H L}, x_{H L}\right)$ belongs to the support of $z(\theta)$ and $x_{H L} \neq V_{M}-y_{H L}$. Let us suppose that only $B$ agents update their characteristics: they will all accept $y_{H L}$. For whatever belief regards the behavior of the opponents, this action is always a best reply. It is then impossible to return to the original state $\theta$. This contradicts the assumption that $\theta \in \Omega$.

Point (ii). First we show that $\Omega$ cannot include a state $\theta$ in which multiple demands are made at $H L$. Subsequently, we show that $\Omega$ cannot include two different states supporting different outcomes following $H L$.

Let $\theta$ be a state such that: (a) $\theta \in \Omega$ and (b) multiple demands are made by agents $A$ at $H L$. We already know from point (i) that at $\theta$ all agents $B$ accept all the demands made by their opponents. Suppose now that only agents $A$ revise their characteristics. Afterward, any agent $A$ will make the maximum 
demand observed at $H L$. Hence, it is impossible to return to the original state $\theta$. This contradicts the assumption that $\theta \in \Omega$.

Now let $\theta$ and $\theta^{\prime}$ be two states such that: (a) both states belong to $\Omega$ and (b) $H L$ is observed. A single demand is made by $A$ but $y_{H L}\left(\theta^{\prime}\right)>y_{H L}(\theta)$. Since it is impossible to return to $\theta$ then assumption $\theta \in \Omega$ is contradicted.

Lemma 14 Let $\Omega$ be a limit set of $(\Theta, P)$. If $\left\{(H H, y, x) ;\left(H H, y^{\prime}, x^{\prime}\right)\right\} \in \rho(\Omega)$ and either $x \neq x^{\prime}$ or $y \neq y$, then $\Omega$ is a singleton and a self-confirming equilibrium of $\Gamma$.

Proof. Consider a set $\Omega$ and let $\theta \in \Omega$ be a state in which at least two demands have been observed in one population (i.e. $B$ ). Suppose that at least one of these demands $\left(x^{*}\right)$ is not a best reply to $z(\theta)$. Suppose also that, after observing $z(\theta)$, all agents who demanded $x^{*}$ revise; as a consequence $x^{*}$ disappears. A new state $\bar{\theta} \in \Omega$ is then reached in which profile $H H$ is still observed. Suppose now that all $A$ update; then, by Lemma 12, nobody will make demand $\left\{V_{H}-x^{*}\right\}$. These two demands have thus disappeared and it is impossible to return to the original state $\theta$. This contradicts the assumption that $\theta \in \Omega$. Therefore, if multiple demands are made, each must be a best reply to $z(\theta)$.

Now consider an agent belonging to population $A$ who has played $H$ in $\theta$ and suppose this agent has the incentive to change her investment should she know $z(\theta)$. When this agent updates, the distribution of the demands made by population $A$ in subgame $H H$ differs from the original. This implies that at least one demand made by some opponents (i.e. $B$ ) is no longer a best reply when $B$ updates. By applying the argument made in the paragraph above, we conclude that at least one pair of demands has disappeared and cannot reappear. This contradicts the assumption that $\theta \in \Omega$.

\section{Proof of Proposition 2}

Assume that $\Omega$ is not a singleton. We know from Lemmas 13 and 14 that, if a bargaining subgame is reached, only one of its terminal nodes is almost always observed.

First we show that $\rho(\Omega)$ must contain one outcome for every bargaining subgame. Of course $\rho(\Omega)$ must differ from $\left\{\left(H H, y_{H H}, V_{H}-y_{H H}\right),(L L, 0,0)\right\}$. Suppose $\rho(\Omega)$ includes the following outcomes: (a) $\left(H H, y_{H H}, x_{H H}\right)$ with $y_{H H}+$ $x_{H H}=V_{H}$; (b) $\left(H L, y_{H L}, x_{H L}\right)$ with $y_{H L}+x_{H L}=V_{M}$. In $\Omega$ a state $\theta$ in which both outcomes are observed must exist and it cannot be an equilibrium. We show that it is possible to reach either the basin of attraction of one equilibrium of the game, or a state in which all bargaining nodes are observed from $\theta$. Suppose some agents $B$ update. If $x_{H H}-c>x_{H L}$ then the updating agents will choose $H$ so that, at the new state $\theta^{\prime}$, the frequency of this action in population $B$ will increase.

If at least one agent $A$ has beliefs $\widetilde{y}_{L H}^{i}$ leading her not to prefer $H$ to $L$ when all agents $B$ play $H$, then it is possible to reach a state in which all 
investment profiles are realized from $\theta$. This contradicts the assumption that $\rho(\Omega)=\left\{\left(H H, y_{H H}, x_{H H}\right) ;\left(H L, y_{H L}, x_{H L}\right)\right\}$. Otherwise, by letting all agents $B$ update from $\theta$ it is likely to reach the basin of attraction of one equilibrium of the game supporting the outcome $\left(H H, y_{H H}, x_{H H}\right)$. If $x_{H H}-c \leq x_{H L}$ we reach the same conclusion by using a similar argument. It is simple to see that the same conclusion holds when $\rho(\Omega)$ includes any two different outcomes. Therefore if $\Omega$ is a not a singleton, all the bargaining nodes are almost always visited meaning that $\rho(\Omega)$ includes four outcomes each of which is a subgame equilibrium.

We now have to show that the payoffs must satisfy a well-defined set of constraints. Notice that a state $\theta \in \Omega$ in which all the investment profiles are observed must exist. Moreover when we allow all agents to update, all agents $A$ will choose $H$ :

$$
p_{B}(\theta)\left(y_{H H}-y_{L H}-c\right)+\left(1-p_{B}(\theta)\right)\left(y_{H L}-c\right)>0,
$$

and all agents $B$ will choose $H$ :

$$
p_{A}(\theta)\left(x_{H H}-x_{H L}-c\right)+\left(1-p_{A}(\theta)\right)\left(x_{L H}-c\right)>0 .
$$

We can rewrite these conditions as

$$
\begin{aligned}
& p_{B}(\theta) A_{1}+\left(1-p_{B}(\theta)\right) A_{2}>0 \\
& p_{A}(\theta) B_{1}+\left(1-p_{A}(\theta)\right) B_{2}>0 .
\end{aligned}
$$

If all expressions are null, then $\Omega$ is a singleton. Furthermore, when - for some populations - both expressions are either not negative or not positive, and at least one is not null, then the process can reach a new state from $\theta$ which is a self-confirming equilibrium.

Consider the case in which both expressions are null for population $A$ only. When $B_{1}$ is strictly positive and $B_{2}$ is strictly negative all $B s$ prefer $H$ if $p_{A}(\theta)>$ $p_{A}^{*}$ where:

$$
p_{A}^{*}=\frac{c-x_{L H}}{\left(x_{H H}-x_{H L}\right)-x_{L H}} .
$$

Otherwise when $B_{1}$ is strictly negative and $B_{2}$ is strictly positive all $B s$ prefer $H$ if $p_{A}(\theta)<p_{A}^{*}$. In both cases, when all $B s$ agents update they will choose the same investment. Hence, a state which is an equilibrium of the game can be reached from $\theta$.

A similar argument is applied when both expressions $B_{1}$ and $B_{2}$ are null. In this case, the threshold value of $p_{B}(\theta)$ is $p_{B}^{*}$ which is now given by:

$$
p_{B}^{*}=\frac{c-y_{H L}}{\left(y_{H H}-y_{L H}\right)-y_{H L}} .
$$


We are left with the case in which the product of the corresponding two expressions is strictly negative for each population. However, when $A_{1}$ and $B_{1}$ have the same sign the process can go into the basin of attraction of an equilibrium. Indeed, suppose that both $A_{1}$ and $B_{1}$ are strictly positive. This implies that all $B s$ prefer $H$ if $p_{A}(\theta)>p_{A}^{*}$ and all $A s$ prefer $H$ if $p_{B}(\theta)>p_{B}^{*}$. Hence, for whatever values of $p_{A}(\theta)$ and $p_{B}(\theta)$, starting from $\theta$ the process can reach an equilibrium when one population revises at a time. The remaining possible case occurs when $B_{1} B_{2}<0$ and $A_{1} A_{2}<0$ but $A_{1} B_{1}<0$.

\section{Proof of Proposition 3}

It follows by applying the same arguments as above and taking into account that $y_{H L}=x_{L H}=V_{M}-\delta$ holds at any limit set. In this case the conditions $B_{1} B_{2}<0, A_{1} A_{2}<0$ and $A_{1} B_{1}<0$ can not be simultaneously met because both $A_{2}$ and $B_{2}$ are strictly positive. Consequently all limit sets are singleton. In addition an equilibrium of the game can only support outcome $(L L, 0,0)$ if it also supports at least one outcome following each investment profile.

Before giving the proof we briefly review the concept of mutation connected set.

Definition 15 Consider a union of limit sets $X$. This set is mutation connected if for all pairs $\Omega, \Omega^{\prime} \in X$ exists a sequence of limit sets $\left(\Omega_{1}=\Omega, \Omega_{2}, \ldots, \Omega_{n}=\Omega^{\prime}\right)$ such that (a) for any $k \in\{1, \ldots, n-1\}, \Omega_{k} \in X$ and (b) every transition from $\Omega_{k}$ to $\Omega_{k+1}$ needs no more than one mutation.

Consider a limit set $\Omega$ which does not support all information sets and suppose a single mutation occurs. If this mutation is a drift then the process reaches a new limit set $\Omega^{\prime}$ which differs from $\Omega$ only for some belief and/or action at some unreached information sets. Let $\Sigma(\Omega)$ be the set of equilibria which only differ from $\Omega$ for some beliefs (and/or actions) held in some unreached information set. Sure enough the set $\Sigma(\Omega)$ is mutation connected. When $\Omega$ is singleton, namely $\Omega=\{\theta\}$, we use $\Sigma(\theta)$ instead of $\Sigma(\Omega)$.

\section{Proof of Lemma 4}

We give the detailed proof for $\Gamma_{U G}$. Then we suggest how to adapt it to $\Gamma_{D G}$. In this proof, when $\theta$ is an equilibrium and multiple demands are observed at $H H$, we denote the full ordered sets of demands made by $B$ and $A$ by $\left\{x_{H H, l}\right\}_{l=1}^{k}$ and $\left\{y_{H H, l}\right\}_{l=1}^{k}$ respectively. By iterative applications of Lemma (12) we get $\left\{y_{H H, l}\right\}_{l=1}^{k}=\left\{V_{H}-x_{H H, l}\right\}_{l=k}^{1}$. Since $\theta$ is an equilibrium then the expected payoff at $H H$ is:

$$
\begin{aligned}
& \widetilde{y}_{H H}^{i}(\theta)=y_{H H, 1}=V_{H}-x_{H H, k} ; \forall i \in A \\
& \widetilde{x}_{H H}^{i}(\theta)=x_{H H, 1}=V_{H}-y_{H H, k} ; \forall i \in B
\end{aligned}
$$

Moreover $y_{H H, 1}=y_{H H, k} \eta_{1}^{B}$ and $x_{H H, 1}=x_{H H, k} \eta_{1}^{A}$ where $\eta_{1}^{B}$ (resp. $\eta_{1}^{A}$ ) is the number of $B$ (resp. $A$ ) agents who claim $x_{H H, 1}\left(\right.$ resp. $\left.y_{H H, 1}\right)$ under $\theta$. 
I) Consider an equilibrium $\theta$ in which only one profile is realized and multiple demands are made at $H H$ profile. Let a single agent $B$ switch from $x_{H H, k}$ to $x_{H H, 1}$. Let all agents $A$ update; then they will make a demand $y_{H, k}=$ $V_{H}-x_{H H, 1}$. Hence, we reach a new equilibrium $\theta^{\prime}$ in which only $H H$ is observed and only the two demands $\left(V_{H}-x_{H H, 1}, x_{H H, 1}\right)$ occur.

II) Suppose now that two profiles are observed at the equilibrium $\theta$. This implies that the same type of investment is made in one population. We give the proof only when $H H$ and $H L$ are observed. The other remaining cases are similar.

II.1) First, consider the case in which multiple demands are made following $H H$. Since $\theta$ is an equilibrium, the following conditions must always be met:

$$
\begin{aligned}
& p_{B}(\theta)\left(y_{H H, 1}-\widetilde{y}_{L H}^{i}(\theta)-c\right)+\left(1-p_{B}(\theta)\right)\left(y_{H L}-c\right) \geq 0, \forall i \in A \\
& \left(V_{H}-y_{H H, k}\right)-c=V_{M}-y_{H L}, \forall i \in B .
\end{aligned}
$$

Consider an equilibrium $\theta_{1} \in \Sigma(\theta)$ in which $\left(y_{H H, 1}-\widetilde{y}_{L H}^{i}\left(\theta_{1}\right)-c\right)>0$ for all $A s$. When $y_{H H, 1}-c>\delta$, the population can get from $\theta$ to $\theta_{1} \in \Sigma(\theta)$ through a sequence of single-mutations. At $\theta_{1}$ let a single agent $A$ mutate from $y_{H H, k}$ to $y_{H H, 1}$ and let all agents $B$ revise; as a consequence they will all choose $H$ and ask $\left(V_{H}-y_{H H, 1}\right)$. Therefore, the process enters a new equilibrium $\theta^{\prime}$ where $\rho\left(\theta^{\prime}\right)=\left\{H H, y_{H H, 1}, V_{H}-y_{H H, 1}\right\}$. Instead, when $y_{H H, 1}-c \leq \delta$, the inequality $y_{H L}-c \geq 0$ must hold for all $A s$. Suppose a single $A$ mutates from $y_{H, k}$ to $\bar{y}$ where $\bar{y}>y_{H H, k}$ and let all agents $B$ update: as a consequence they all will choose $L$. Therefore, by a sequence of single mutations the process reaches a new equilibrium $\theta^{\prime}$ where $\rho\left(\theta^{\prime}\right)=\left\{H L, y_{H L}, V_{M}-y_{H L}\right\}$.

II.2) Now consider the case in which a single demand is made following $H H$. When $y_{H L}-c \geq 0$, the process can reach a new equilibrium $\theta^{\prime}$ where $\rho\left(\theta^{\prime}\right)=\left\{H L, y_{H L}, V_{M}-y_{H L}\right\}$ by letting a single agent $A$ mutate from $y_{H H, 1}$ to $\bar{y}$ where $\bar{y}>y_{H H, 1}$ and all agents $B$ to revise. When instead $y_{H L}-c<0$, then: (a) since point (c) of Assumption 1 holds, the subgame $\left(H L, V_{M}-\delta\right)$ at $\theta$ is not reached; (b) $y_{H H, 1}-\widetilde{y}_{L H}^{i}\left(\theta_{1}\right)-c \geq 0$ for every $A$. By drifting, all agents $B$ are led to accept the maximum feasible demand made by $A$ in $H L$ so that a new equilibrium $\theta_{1}$ is reached. Sure enough, $\theta_{1} \in \Sigma(\theta)$. Suppose now that a single agent $A$ changes her demand from $y_{H L}$ to $\left(V_{M}-\delta\right)$. When all agents $A$ update, they observe that all $B s$ have accepted the demand $\left(V_{M}-\delta\right)$; therefore, in $H L$ their best response is $y_{H L}=V_{M}-\delta$. When all agents $B$ update, they will choose $H$ being $x_{H L}=\delta$. Hence, the process reaches equilibrium $\theta^{\prime}$ where $\rho\left(\theta^{\prime}\right)=\left\{H H, y_{H H, 1}, V_{H}-y_{H, 1}\right\}$.

III) Suppose now that all investment profiles are observed at equilibrium $\theta$. Since $\theta$ is an equilibrium the following conditions must be satisfied:

$$
\begin{aligned}
& p_{B}(\theta)\left(y_{H H, 1}-y_{L H}-c\right)+\left(1-p_{B}(\theta)\right)\left(y_{H L}-c\right)=0 \\
& p_{A}(\theta)\left(x_{H H, 1}-x_{H L}-c\right)+\left(1-p_{A}(\theta)\right)\left(x_{L H}-c\right)=0 .
\end{aligned}
$$


where $y_{H H, 1}=V_{H}-x_{H H, k}, y_{H L}=V_{M}-x_{H L}$ and $y_{L H}=V_{M}-x_{L H}$. We may rewrite these conditions as

$$
\begin{aligned}
& p_{B}(\theta) A_{1}^{\prime}+\left(1-p_{B}(\theta)\right) A_{2}^{\prime}=0 \\
& p_{A}(\theta) B_{1}^{\prime}+\left(1-p_{A}(\theta)\right) B_{2}^{\prime}=0 .
\end{aligned}
$$

We argue that when at least one of the expressions $A_{2}^{\prime}$ and $B_{2}^{\prime}$ is not positive then the process, through a sequence of single-mutations, can reach one equilibrium in which a smaller number of investment profiles are realized. In order to see this suppose, for instance, that $A_{2}^{\prime}<0$; then, under assumption (2) at $\theta$ the subgame $\left(H L, V_{M}-\delta\right)$ is not reached. A drift can lead all agents $B$ to accept the maximum feasible demand of the opponent at $H L$. A new $\theta_{1} \in \Sigma(\theta)$ is then reached. Suppose now that at this new equilibrium a single agent $A$ mutates her demand from $y_{H L}$ to $V_{M}-\delta$. When all agents $A$ revise, they will play $H$ and will make a demand $y_{H L}=V_{M}-\delta$. Now let all agents $B$ update. Since each agent $B$ knows that $x_{H L}=\delta$ and that all $A s$ have played $H$, then her best reply depends on the sign of $\left(x_{H H, 1}-\delta-c\right)$. However, it is simple to see that for whatever value of $\left(x_{H H, 1}-\delta-c\right)$, the process can reach a new equilibrium in which a smaller number of investment profiles is realized. If, at this new equilibrium, two investment profiles are realized, then the process can reach an equilibrium which supports a single outcome by a further sequence of single transition (see point II.2 above).

When both $A_{2}^{\prime}$ and $B_{2}^{\prime}$ are positive, a single mutation occurring in population $A$ is enough to trigger a shift from $\theta$ to a new equilibrium $\theta^{\prime}$ where:

$$
\rho\left(\theta^{\prime}\right)=\left\{L H, y_{L H}, V_{M}-y_{L H}\right\} .
$$

In this case the mutation needed depends on how many demands are observed at $H H$. In particular:

(i) when multiple demands are made at $H H$, it is enough for one agent $A$ to mutate from $y_{H H, k}$ to $y_{H H, 1}$;

(ii) when only one demand is made at $H H$ it is enough for one agent $A$ to mutate from $H$ to $L$;

IV) The remaining case occurs when $\Omega$ is not a singleton. Under the assumption (2) at least one of the following two subgames $\left(L H, V_{M}-\delta\right)$ and $\left(H L, V_{M}-\delta\right)$ is never reached. The same argument used above implies that the population can get from $\Omega$ to $\theta^{\prime}$ through a sequence of single-mutations.

Now consider game $\Gamma_{D G}$. Some of the arguments above continue to work with minor modifications. First of all, notice that $y_{H L}=x_{L H}=V_{M}-\delta$ in this case. Therefore, it follows that: (a) in case II) the set of investments profiles supported by an equilibrium can either be $\{H H, H L\}$ or $\{H H, L H\}$; (b) in case III) both $A_{2}^{\prime}$ and $B_{2}^{\prime}$ are positive; (c) case IV) does not arise.

\section{Proof of Lemma 5}


Since in $\Gamma_{D G}$ the set $\Sigma_{L}$ is empty, the first point of the lemma hold only for $\Gamma_{U G}$ •

(a) Let $\theta$ be an equilibrium belonging to $\Sigma_{L}$. From $\theta$, by a sequence of single mutations, the process reaches a new equilibrium $\theta^{*} \in \Sigma(\theta)$ in which for every agent $A$ and $B$ it is true that: (i) $\widetilde{y}_{H H}^{i}\left(\theta^{*}\right)=V_{H}-x_{H H}$ and $\left(V_{H}-x_{H H}\right)>c+\delta$; (ii) at the subgame $\left(L H, V_{M}-\delta\right)$ each agent $A$ accepts (i.e. she chooses $\delta$ ); (iii) $\widetilde{x}_{H L}^{i}\left(\theta^{*}\right)=\delta$ and $\widetilde{x}_{H H}^{i}\left(\theta^{*}\right)=x_{H H}$ and $x_{H H}-\delta-c \geq 0$. Suppose now, that an agent $B$ mutates by playing $H$ and makes a demand $V_{M}-\delta$ in $L H$. When all agents $B$ update, they will choose $H$ since all agents $A$ have accepted demand $V_{M}-\delta$. Suppose now that all agents $A$ revise. Since $\left(V_{H}-x_{H H}\right)>c+\delta$, they will play $H$. Hence, the process reaches a new equilibrium $\theta^{\prime} \in \Sigma_{H}$ where $\rho\left(\theta^{\prime}\right)=\left\{H H,\left(V_{H}-x_{H H}\right), x_{H H}\right\}$.

(b) Consider $\Gamma_{U G}$ and let $\theta$ be an equilibrium belonging to $\Sigma_{H L}$. At $\theta$ the pair of demands $\left(y_{H L}, V_{M}-y_{H L}\right)$ is observed. Suppose that $y_{H L}<V_{M}-\delta$. By drifting, all agents $B$ are led to accept the maximum feasible demand made by $A$ in $H L$ and deem that all $A$ make demand larger than $\left(V_{H}-c+\delta\right)$ at $H H$. A new equilibrium $\theta_{1}$ is thus reached. Sure enough, $\theta_{1} \in \Sigma(\theta)$. Suppose now that a single agent $A$ changes her demand from $y_{H L}$ to $\left(V_{M}-\delta\right)$. When all agents $A$ update, they observe that all $B s$ have accepted the demand $\left(V_{M}-\delta\right)$; therefore, in $H L$ their best response is $y_{H L}=V_{M}-\delta$. When all agents $B$ update, since $\widetilde{x}_{H H}^{i}\left(\theta_{1}\right)<c+\delta$ holds for all $B s$, they continue to play $L$. Hence, the process reaches a new equilibrium $\theta^{\prime} \in \Sigma_{H L}$ where $\rho\left(\theta^{\prime}\right)=\left\{H L,\left(V_{M}-\delta\right), \delta\right\}$. From $\theta^{\prime}$, by a sequence of single mutations, the process can reach an equilibrium $\theta^{*} \in \Sigma\left(\theta^{\prime}\right)$ in which all agents $A$ have beliefs such that: (i) $\widetilde{y}_{H H}^{i}\left(\theta^{*}\right)=y_{H H}$; (ii) $\widetilde{y}_{L H}^{i}\left(\theta^{*}\right)=\delta$; (iii) $\delta+c<y_{H H}<V_{H}-c-\delta$. Suppose now, that an agent $B$ mutates by playing $H$ and making a demand $\left(V_{H}-y_{H H}\right)$ in $H H$. Let all agents $B$ revise; they will choose $H$ and ask $\left(V_{H}-y_{H H}\right)$. When agents $A$ update, the process reaches a new equilibrium $\theta^{\prime} \in \Sigma_{H}$ in which the pair of demands is $\left(y_{H H}, V_{M}-y_{H H}\right)$. Of course, if $y_{H L}=V_{M}-\delta$ then only the last sequence of mutations is required. The same argument holds true for $\Gamma_{D G}$ with the caveat that any equilibrium belonging to $\Sigma_{H L}$ supports the outcome $\left(V_{M}-\delta, \delta\right)$ only.

\section{Proof of Lemma 6}

Let $\theta$ be an equilibrium belonging to $\Sigma_{L}$. From $\theta$, by a sequence of single mutations, the process can reach a new equilibrium $\theta^{*} \in \Sigma(\theta)$ in which, for every agent $A$ it is true that at the subgame $\left(L H, V_{M}-y_{L H}\right)$ each agent $A$ accepts (i.e. she chooses $\left.y_{L H}\right)$ and $\widetilde{y}_{H H}^{i}\left(\theta^{*}\right)-y_{L H}-c<0$ but $\left(V_{M}-y_{L H}\right)-c>0$. Suppose now, that an agent $B$ mutates by playing $H$ and makes a demand $V_{M}-y_{L H}$ in $L H$. When all agents $B$ update, they will choose $H$ since population $A$ has accepted demand $V_{M}-y_{L H}$. Suppose now that all agents $A$ revise. Since $\widetilde{y}_{H H}^{i}\left(\theta^{*}\right)-y_{L H}-c<0$, they will continue to play $L$. Hence the process reaches a new equilibrium $\theta^{\prime} \in \Sigma_{L H}$ in which the pair of demands is $\left(y_{L H}, V_{M}-y_{L H}\right)$

\section{Proof of Lemma 8}


Point (a). Consider some $\theta \in \Sigma_{I H}$ and let $\left\{V_{H}-x, x\right\}$ be the observed pair of demands. Note that $\Sigma_{I H}$ is always well defined under condition (6). We show that a single mutation transition is not enough to push the process into the basin of attraction of a different equilibrium which does not belong to $\Sigma_{I H}$.

I) First of all we show that a single mutation from $H$ to $L$ does not enable the process to enter the basin of attraction of a different equilibrium, even if each agent expects to receive: (i) the maximum payoff when she plays $L$ but the opponent still plays $H$; (ii) the minimum payoff when she plays $H$ but the opponent shifts to $L$. Let us consider this scenario and suppose that a $B$ agent had switched from $H$ to $L$ and all agents $A$ revised. This updating does not cause agents $A$ to play $L$ if

$$
\frac{N-1}{N}\left[\left(V_{H}-x-c\right)-\left(V_{M}-\delta\right)\right]+\frac{1}{N}(\delta-c)>0
$$

which can be rewritten as

$$
\frac{N-1}{N}\left(V_{H}-x\right)-c>\frac{N-1}{N} V_{M}-\delta .
$$

Since $x \in\left[\widehat{x}_{B}, \widehat{x}_{A}\right]$ then condition (16) holds by definition. Therefore no agent $A$ will change her action after the revision. Similar argument can be applied to population $B$. Hence, a single mutation from $H$ to $L$ does not trigger a transition to a different equilibrium.

II) We now show that a single mutation from $x$ to $x^{\prime}$ (resp. from $V_{H}-x$ to $y^{\prime}$ ) does not enable the process to enter the basin of attraction of a different equilibrium. Suppose each agent expects to get the maximum payoff when she plays $L$ and the opponent chooses $H$. Let one agent $B$ only change her demand to $x^{\prime}$. Obviously, no agents $B$ imitate the mutant when revising. Consider population $A$ and allow them to update. By Lemma (12) their best response is either $\left(V_{H}-x\right)$ or $\left(V_{H}-x^{\prime}\right)$.

If $x^{\prime}>x$, agent $A$ expects to receive $\left(V_{H}-x\right) \frac{N-1}{N}-c$ when she demands $\left(V_{H}-x\right)$ and $\left(V_{H}-x^{\prime}\right)-c$ when she demands $\left(V_{H}-x^{\prime}\right)$. It is simple to see that under Assumption (3), the former payoff is greater than the latter. Hence, agents $A$ will not change their demand when updating. Moreover, since $\left(V_{H}-x\right) \frac{N-1}{N}-$ $c \geq\left(V_{M}-\delta\right)$, then updating will not cause agents $A$ to play action $L$.

If $x^{\prime}<x$, agent $A$ expects to get $\left(V_{H}-x\right)-c$ when she demands $\left(V_{H}-x\right)$ and $\frac{1}{N}\left(V_{H}-x^{\prime}\right)-c$ when she demands $\left(V_{H}-x^{\prime}\right)$. It is simple to see that under Assumption (3) the former payoff is greater than the latter. Hence, agents $A$ will not change their demand when updating. Moreover, since $\left(V_{H}-x\right)-c>$ $\left(V_{M}-\delta\right)$, then updating will not cause agents $A$ to play action $L$. The case in which an agent $A$ mutates from $V_{H}-x$ to $y^{\prime}$ is symmetric. Hence, a single mutation from $x$ to $x^{\prime}$ (resp. from $V_{H}-x$ to $y^{\prime}$ ) does not trigger a transition to a different equilibrium.

Points I) and II) taken together say that, whatever single mutation we consider, this does not trigger a transition from $\theta \in \Sigma_{I H}$ to a different equilibrium 
outside the set $\Sigma(\theta)$. Indeed, the population returns to equilibrium $\theta^{\prime} \in \Sigma(\theta)$ as soon as the mutating agent revises.

Point (b). Consider some $\theta \in \Sigma_{C H}$ and let $\left\{V_{H}-x, x\right\}$ be the observed pair of demands. We show that a single mutation transition is enough to enter the basin of attraction of an equilibrium $\theta^{\prime}$ belonging to $\Sigma_{L}$. In order to fully describe the transition from $\theta$ to $\theta^{\prime}$, we have to take four cases into account : (1) $x>x_{A}^{*}$; (2) $x=x_{A}^{*}$; (3) $x<x_{B}^{*}$; (4) $x=x_{B}^{*}$. Since cases (3) and (4) are symmetric to case (1) and case (2) respectively, we will give proof for these latter cases only.

Case (1): $x>x_{A}^{*}$.

At $\theta$ the following inequality must hold:

\begin{tabular}{ll} 
Population $A$ & Population $B$ \\
\hline$V_{H}-x-c-\widetilde{y}_{L H}^{i}(\theta) \geq 0$ & $x-c-\widetilde{x}_{H L}^{i}(\theta) \geq 0$ \\
$V_{H}-x-c<V_{M}-\delta$ & $x-c>V_{M}-\delta$.
\end{tabular}

From $\theta$ the process can reach a new equilibrium $\theta_{1} \in \Sigma(\theta)$ by a sequence of single mutations, in which the following is true for every agent: (i) $\widetilde{x}_{L H}^{i}\left(\theta_{1}\right)=\delta$; (ii) $\widetilde{y}_{H L}^{i}\left(\theta_{1}\right)-c<0$. Suppose an agent $A$ mutates by playing $L$ and accepting the demand made by her opponent at $L H$. Let all agents $A$ update. Since the mutating agent receives $V_{M}-\delta$, all $A s$ imitate and play $L$. When agents $B$ revise they will play $L$. The process then reaches a new equilibrium $\theta^{\prime} \in \Sigma_{L}$.

Case (2): $x=x_{A}^{*}$.

At $\theta$ the following inequality must hold for agents $A$ :

$$
V_{H}-x_{A}^{*}-c=V_{M}-\delta
$$

From $\theta$, the process can reach a new equilibrium $\theta_{1} \in \Sigma(\theta)$ by a sequence of single mutations, in which the following it is true for every agent: (i) $\widetilde{x}_{L H}^{i}\left(\theta_{1}\right)=$ $x_{L H}$; (ii) $x_{L H}-c<0$; (iii) $\widetilde{y}_{L H}^{i}\left(\theta_{1}\right)=\left(V_{M}-\delta\right)$; (iv) $\widetilde{y}_{H L}^{i}\left(\theta_{1}\right)-c<0$. Suppose an agent $B$ mutates by demanding $x^{\prime}>x_{A}^{*}$ at $H H$. When agents $A$ update they will all choose $L$ since, for whatever best action at $H H$, the expected payoff by playing $H$ is now smaller than $V_{M}-\delta$. When all agents $B$ revise they will play $L$. The process then reaches a new equilibrium $\theta^{\prime} \in \Sigma_{L}$.

Point (c). Under condition (6) $\Sigma_{I H}$ is well defined. By a direct application of previous point (b), along with point (e) of Lemma 5, it follows that from $\theta \in \Sigma_{C H}$ it is possible to reach $\widehat{\theta} \in \Sigma_{I H}$ through a sequence of single mutations.

The following two Lemmas are needed for the proof of Proposition 9. In the text below we write $\theta_{x}$ as shorthand for an equilibrium belonging to $\Sigma_{I H}$ with $\left(V_{H}-x, x\right)$ as distributional rule. 
Lemma 16 Consider $\Gamma_{U G}$ and let $\theta_{x} \in \Sigma_{I H}$. The minimum number of mutations required to get from $\Sigma\left(\theta_{x}\right)$ to an equilibrium which supports a different investment profile is:

$$
\begin{aligned}
& \bar{r}_{A}(x)=\left\lfloor N\left(1-\frac{V_{M}-\delta+c}{x}\right)\right\rfloor \quad \text { if } \quad x<\frac{V_{H}}{2} \\
& \bar{r}_{B}(x)=\left\lfloor N\left(1-\frac{V_{M}-\delta+c}{V_{H}-x}\right)\right\rfloor \quad \text { if } \quad x>\frac{V_{H}}{2}
\end{aligned}
$$

where $\lfloor s\rceil$ denotes the least integer greater than $s$ when $s$ is not an integer and $(s+1)$ is otherwise.

Proof. Suppose $p_{1}$ agents $B$ mutate by playing $L$ and $p_{2}$ agents $B$ mutate by claiming $x^{\prime}>x_{A}^{*}$. For a given pair $\left(p_{1}, p_{2}\right)$, agents $A$ have the largest incentive to change into $L$ if their beliefs are such that: (i) they expect to get the maximum payoff in an $L H$ match ; (ii) they expect to obtain the minimum payoff in an $H L$ match . Consider equilibrium $\widetilde{\theta}_{x} \in \Sigma\left(\theta_{x}\right)$, in which the following applies for all agents:(i); $\widetilde{y}_{L H}^{i}=V_{M}-\delta$ and $\widetilde{y}_{H L}^{i}=\delta$; (ii) $\widetilde{x}_{L H}^{i}=\delta$ and in the subgame $\{H L, \delta\}$ all agents $B$ accept. When at $\widetilde{\theta}_{x}$, some agents $B$ mutate and these mutations induce all agents $A$ to play $L$, then the process enters the basin of attraction of the equilibrium $\theta^{\prime} \in \Sigma_{L}$ with positive probability. Sure enough after updating all agents $A$ decide to play $L$ if

$$
\frac{N-p_{1}}{N}\left(V_{M}-\delta\right)>\mu_{H}\left(\widetilde{\theta}_{x}, p_{1}, p_{2}\right)
$$

where LHS is the expected payoff by playing $L$ and RHS is the expected payoff by playing $H$. However, $\mu_{H}\left(\widetilde{\theta}_{x}, p_{1}, p_{2}\right)$ depends on what the best demand in a match $H H$ is. In particular

$\mu_{H}()=.\left\{\begin{array}{ccc}\frac{N-p_{2}-p_{1}}{N}\left(V_{H}-x\right)+\frac{p_{1}}{N} \delta-c & \text { if } & \frac{N-p_{2}-p_{1}}{N-p_{1}}\left(V_{H}-x\right) \geq\left(V_{H}-x^{\prime}\right) \\ \frac{N-p_{1}}{N}\left(V_{H}-x^{\prime}\right)+\frac{p_{1}}{N} \delta-c & \text { if } & \frac{N-p_{2}-p_{1}}{N-p_{1}}\left(V_{H}-x\right)<\left(V_{H}-x^{\prime}\right)\end{array}\right.$

The minimum number of mutations in population $B$ comes from the comparison between the solutions of two constrained minimization problems. In both problems, the objective function is $p_{1}+p_{2}$. In the first (resp. second) problem we contemplate the case in which the best action in $H H$ is $V_{H}-x^{\prime}\left(\operatorname{resp} . V_{H}-x\right)$. Both problems require $p_{1}=0$ as a solution. Moreover, $p_{2}^{M 1}=N\left(\frac{x^{\prime}-x}{V_{H}-x}\right)$ is the solution to the first problem and $p_{2}^{M 2}=N\left(1-\frac{V_{M}-\delta+c}{V_{H}-x}\right)$ is the solution to the second. Since $p_{2}^{M 1}>p_{2}^{M 2}$, the minimum number of mutations in population $B$ involves that: (i) mutating agents only change their demands in the $H H$ profile; (ii) these mutations cause agent $A$ to shift to action $L$ when the best action in the $H H$ match continues to be $\left(V_{H}-x\right)$. Hence:

$$
\bar{r}_{B}(x)=\left\lfloor N\left(1-\frac{V_{M}-\delta+c}{V_{H}-x}\right)\right\rceil
$$


and

$$
\bar{r}_{B}=\min _{x} \bar{r}_{B}(x)=\bar{r}_{B}\left(\widehat{x}_{A}\right) .
$$

We now suppose that some agents $A$ mutate. As before, two kinds of mutations must be considered: $p_{1}$ agents $A$ mutate by playing $L$ and $p_{2}$ agents $A$ mutate by demanding $\left(V_{H}-x^{\prime}\right)$ where $x^{\prime}<x_{B}^{*}$. In this case, we look for an equilibrium $\widehat{\theta}_{x} \in \Sigma\left(\theta_{x}\right)$ in which for all agents: (i) $\widetilde{x}_{L H}^{i}=\delta$ and $\widetilde{x}_{H L}^{i}=V_{M}-\delta$; (ii) $\widetilde{y}_{H L}^{i}=\delta$ and in the subgame $\{L H, \delta\}$ all agents $A$ accept. It is easy to see that if some mutations of agents $A$ occurs at $\widehat{\theta}_{x}$ and these mutations induce all agents $B$ to play $L$, then the process enters the basin of attraction of equilibrium $\theta^{\prime} \in \Sigma_{L}$ with positive probability.

After updating, all agents $B$ decide to play $L$ if

$$
\frac{N-p_{1}}{N}\left(V_{M}-\delta\right)>\mu_{H}\left(\widehat{\theta}_{x}, p_{1}, p_{2}\right)
$$

where

$$
\mu_{H}(.)=\left\{\begin{array}{lll}
\frac{N-p_{2}-p_{1}}{N} x+\frac{p_{1}}{N} \delta-c & \text { if } & \frac{N-p_{2}-p_{1}}{N-p_{1}} x \geq x^{\prime} \\
\frac{N-p_{1}}{N} x^{\prime}+\frac{p_{1}}{N} \delta-c & \text { if } & \frac{N-p_{2}-p_{1}}{N-p_{1}} x<x^{\prime} .
\end{array}\right.
$$

Proceeding as before, the minimum number of mutations in population $A$ is

$$
\bar{r}_{A}(x)=\left\lfloor N\left(1-\frac{V_{M}-\delta+c}{x}\right)\right\rceil
$$

and

$$
\bar{r}_{A}=\min _{x} \bar{r}_{A}(x)=\bar{r}_{A}\left(\widehat{x}_{B}\right)
$$

By comparing (22) and (25) we obtain $\bar{r}_{B}(x)<\bar{r}_{A}(x)$ if $x>\frac{V_{H}}{2}$.

Lemma 17 For $\delta$ sufficiently small, the minimum number of mutations needed to get from $\Sigma\left(\theta_{x}\right)$ to an equilibrium with the same investment profile but different demands is:

$$
\begin{aligned}
& r_{B}^{+}(x)=\left\lfloor N\left(\frac{\delta}{V_{H}-x}\right)\right\rceil \quad \text { if } \quad x<\frac{V_{H}}{2} \\
& r_{A}^{-}(x)=\left\lfloor N\left(\frac{\delta}{x}\right)\right\rceil \quad \text { if } \quad x>\frac{V_{H}}{2}
\end{aligned}
$$

where $r_{B}^{+}(x)$ is the number of mutations needed for the transition from $\Sigma\left(\theta_{x}\right)$ to $\Sigma\left(\theta_{x+\delta}\right)$ whereas $r_{A}^{-}(x)$ is the number of mutations needed for the transition from $\Sigma\left(\theta_{x}\right)$ to $\Sigma\left(\theta_{x-\delta}\right)$. Moreover, $r_{B}^{+}(x)$ is a strictly increasing function of $x$ and $r_{A}^{-}(x)$ is a strictly decreasing function of $x$.

Proof: By a direct application of Young (1993).

\section{Proof of Proposition 9}

Before giving proof we will briefly introduce the radius modified coradius criterion (Ellison, 2000). Let $\Sigma$ be a union of limit sets $(\Omega)$. Radius $R(\Sigma)$ is the 
minimum number of mutations needed to escape from the basin of attraction of $\Sigma$ and enter into the basin of attraction of another one with positive probability. Consider an arbitrary state $\theta \notin \Sigma$ and let $\left(z_{1}, z_{2}, . ., z_{T}\right)$ be a path from $\theta$ to $\Sigma$ where $\Omega_{1}, \Omega_{2}, . . \Omega_{r}$ is the sequence of limit sets through which the path passes consecutively. Obviously $\Omega_{i} \notin \Sigma$ for $i<r$ and $\Omega_{r} \subset \Sigma$. Furthermore, it may be that a limit set can appear several times but not consecutively. The modified cost of this path is defined by:

$$
c^{*}\left(z_{1}, \ldots, z_{T}\right)=c\left(z_{1}, . ., z_{T}\right)-\sum_{i=2}^{r-1} R\left(\Omega_{i}\right)
$$

where $c\left(z_{1}, . ., z_{T}\right)$ is the total number of mutations over the path $\left(\theta, z_{1}, z_{2}, . ., z_{T}\right)$. Let $c^{*}(\theta, \Sigma)$ be the minimal modified cost for all paths from $\theta$ to $\Sigma$. The modified coradius of the basin of attraction of $\Sigma$ is then:

$$
C R^{*}(\Sigma)=\max _{\theta \notin \Sigma} c^{*}(\theta, \Sigma) .
$$

Theorem 2 of Ellison (2000) shows that every union of limit sets $\Sigma$ with $R(\Sigma)>$ $C R^{*}(\Sigma)$ encompasses all stochastically stable states.

From Lemmas 4, 5 and 8, points (b) and (c), we can deduce that for any $\Omega \notin \Sigma_{I H}$ the minimal modified cost for all paths from $\theta$ to $\Sigma_{I H}$, is equal to one, whatever the number of limit sets the path goes through may be. Therefore, $C R^{*}\left(\Sigma_{I H}\right)=1$. Since we know from point (a) of Lemma 8 that $R\left(\Sigma_{I H}\right)>1$, by direct application of Ellison's result it follows that all stochastically stable states are included in $\Sigma_{I H}$.

In order to find the stochastically stable outcome, we need: (i) the radius of $\Sigma(\theta)$, i.e. the smallest number of mutations required to destabilize the outcome supported by $\theta, \forall \theta \in \Sigma_{I H}$; (ii) to find an equilibrium belonging to $\Sigma_{I H}$ such that $R(\Sigma(\theta))>C R^{*}(\Sigma(\theta))$. All the details needed to compute $R\left(\Sigma\left(\theta_{x}\right)\right)$ for every $x \in\left[\widehat{x}_{B} ; \widehat{x}_{A}\right]$ are given by Lemmas 16 and 17 . In particular, Lemma 16 provides the minimum number of mutations required to make a transition from $\theta \in \Sigma_{I H}$ to $\theta^{\prime} \notin \Sigma_{I H}$. Lemma 17 provides the minimum number of mutations required to make a transition from $\theta \in \Sigma_{I H}$ to another equilibrium supporting the same investment profile $H H$, but a different distributional rule. Remember that for any $x \in\left[\widehat{x}_{B} ; \widehat{x}_{A}\right]$ it is always true that

$$
\left(V_{H}-(x+\delta)\right)-c \geq V_{M}-\delta
$$

and

$$
(x-\delta)-c \geq V_{M}-\delta .
$$

Thus, from (28) and (29a) respectively we infer that:

$$
\begin{aligned}
& r_{B}^{+}(x) \leq \bar{r}_{B}(x) \\
& r_{A}^{-}(x) \leq \bar{r}_{A}(x) .
\end{aligned}
$$


For any $x \in\left[\widehat{x}_{B} ; \widehat{x}_{A}\right]$, it follows from Lemma (16) and Lemma (17) that

$$
\begin{aligned}
& R\left(\Sigma\left(\theta_{x}\right)\right)=r_{B}^{+}(x) \quad \text { if } \quad x<\frac{V_{H}}{2} \\
& R\left(\Sigma\left(\theta_{x}\right)\right)=r_{A}^{-}(x) \quad \text { if } \quad x>\frac{V_{H}}{2} .
\end{aligned}
$$

Let $\bar{x} \equiv \frac{V_{H}}{2}$ and consider the set of equilibria $\Sigma\left(\theta_{\bar{x}}\right)$. Let $\theta_{x} \in \Sigma_{I H}$ be an equilibrium. When $x<\bar{x}$, then the minimal modified $\operatorname{cost}$ from $\theta_{x}$ to $\Sigma\left(\theta_{\bar{x}}\right)$ is associated with the path $\theta_{x} \rightarrow \theta_{x+\delta} \rightarrow \ldots \rightarrow \theta_{\bar{x}-\delta} \rightarrow \Sigma\left(\theta_{\bar{x}}\right)$. Conversely, when $x>\bar{x}$, the minimal modified costs is associated with path $\theta_{x} \rightarrow \theta_{x-\delta} \rightarrow \ldots \rightarrow$ $\theta_{\bar{x}+\delta} \rightarrow \Sigma\left(\theta_{\bar{x}}\right)$. Hence

$$
\begin{aligned}
& c^{*}\left(\theta_{x} ; \Sigma\left(\theta_{\bar{x}}\right)\right)=r_{B}^{+}(x) \quad \text { if } \quad x<\frac{V_{H}}{2} \\
& c^{*}\left(\theta_{x} ; \Sigma\left(\theta_{\bar{x}}\right)\right)=r_{A}^{-}(x) \quad \text { if } \quad x>\frac{V_{H}}{2} .
\end{aligned}
$$

By the monotonicity of $r_{B}^{+}(x)$ and $r_{A}^{-}(x)$ we obtain

$$
C R^{*}\left(\Sigma\left(\theta_{\bar{x}}\right)\right)=\max \left(r_{B}^{+}(\bar{x}-\delta) ; r_{A}^{-}(\bar{x}+\delta)\right) .
$$

Since

$$
R\left(\Sigma\left(\theta_{\bar{x}}\right)\right)=r_{B}^{+}(\bar{x})=r_{A}^{-}(\bar{x})>C R^{*}\left(\Sigma\left(\theta_{\bar{x}}\right)\right)
$$

it follows from Ellison (2000) that the only stochastically stable outcome is $\left\{H H, \frac{V_{H}}{2}, \frac{V_{H}}{2}\right\}$.

\section{Proof of Lemma 10}

Consider an equilibrium $\theta \in \Sigma_{H}$ which satisfies condition (7). This implies that:

$$
\begin{gathered}
\left(V_{H}-x\right) \frac{N-1}{N}-c>\delta \\
x \frac{N-1}{N}-c>\delta .
\end{gathered}
$$

Suppose that an agent $A$ (resp. $B$ ) mutates by drifting her belief at $L H$ (resp. $H L)$ to $\widetilde{y}_{L H}>V_{H}-x-c\left(\right.$ resp. $\left.\widetilde{x}_{H L}>x-c\right)$. Under this belief the mutant chooses $L$. This implies that profile $L H$ (resp. $H L$ ) is reached. Then suppose all agents $A$ (resp. $B$ ) revise. Since all agents $B$ (resp. $A$ ) have claimed $\left(V_{M}-\delta\right)$, then for all agents $A$ (resp. $\left.B\right)$ we get $\widetilde{y}_{L H}^{i}=\delta\left(\right.$ resp. $\left.\widetilde{x}_{H L}^{i}=\delta\right)$; hence all agents $A$ (resp. $B$ ) choose to play $H$. The process then returns to an equilibrium $\theta^{\prime} \in \Sigma(\theta)$.

Suppose that a single mutation occurs from $x$ to $x^{\prime}$. Obviously no agent $B$ imitates the mutant when updating. Allow all agents $A$ to revise. By Lemma 12 their best response is either $V_{H}-x$ or $V_{H}-x^{\prime}$.

When $x^{\prime}>x$, agent $A$ expects to receive $\left(V_{H}-x\right) \frac{N-1}{N}-c$ by claiming $V_{H}-x$ and $V_{H}-x^{\prime}-c$ by asking $V_{H}-x^{\prime}$. Otherwise, when $x^{\prime}<x$, agent $A$ expects to receive $V_{H}-x-c$ by claiming $V_{H}-x$ and $\left(V_{H}-x^{\prime}\right) \frac{1}{N}-c$ by asking $V_{H}-x^{\prime}$. It is simple to see that, whatever the relation between $x$ and $x^{\prime}$ is, under assumption 
(3) the best response continues to be $V_{H}-x$. Since $\left(V_{H}-x\right) \frac{N-1}{N}-c>\delta$ then updating will not cause agents $A$ to change investment action.

A few definitions are necessary before giving the proof of Proposition 11; in particular, let

$$
\begin{aligned}
\widehat{x}_{B}^{D} & =\min \left\{x \in D_{\delta}\left(V_{H}\right) \mid x \frac{N-1}{N}-c \geq \delta\right\} \\
\widehat{x}_{A}^{D} & =\max \left\{x \in D_{\delta}\left(V_{H}\right) \mid\left(V_{H}-x\right) \frac{N-1}{N}-c \geq \delta\right\}
\end{aligned}
$$

and let $\Sigma_{I H}^{D}=\left\{\theta \in \Sigma_{H} \mid x \in\left[\widehat{x}_{B}^{D}, \widehat{x}_{A}^{D}\right]\right\}$. Under assumption (3) it follows that $\widehat{x}_{B}^{D}=2 \delta+c$ and $\widehat{x}_{A}^{D}=V_{H}-2 \delta-c$. Therefore the set $\Sigma_{I H}^{D}$ is well-defined only when $\widehat{x}_{B}^{D}<\widehat{x}_{A}^{D}$ which can be translated into condition (8), i.e.

$$
\delta<\frac{V_{H}-2 c}{4} .
$$

Since $V_{H}-2 c>0$ by assumption, then it is always possible to detect a decreasing sequence of $\delta$ which satisfies condition (8). This assures that the set $\Sigma_{I H}^{D}$ is always well-defined in $\Gamma_{D G}$. Moreover, since for all equilibria $\theta \in \Sigma_{I H}^{D}$ condition (7) is satisfied, then Lemma (10) holds.

The following Lemma, which replaces Lemma 16, is needed for the proof of Proposition 11. As before, by $\theta_{x}$ we denote an equilibrium belonging to $\Sigma_{I H}^{D}$ with $\left(V_{H}-x, x\right)$ as the distributional rule.

Lemma 18 Consider $\Gamma_{D G}$ and let $\theta_{x} \in \Sigma_{I H}^{D}$. The minimum number of mutations required to get from $\Sigma\left(\theta_{x}\right)$ to an equilibrium which supports a different investment profile is:

$$
\begin{aligned}
& \bar{r}_{A}(x)=\left[N\left(1-\frac{\delta+c}{x}\right)\right] \\
& \bar{r}_{B}(x)=\left[N\left(1-\frac{\delta+c}{V_{H}-x}\right)\right]
\end{aligned}
$$

where $[s]$ denotes the least integer greater than $s$ when $s$ is not an integer and $(s+1)$ is otherwise.

Proof. We give proof for $\bar{r}_{B}(x)$ only. Similar arguments can be used for $\bar{r}_{A}(x)$. Consider $\theta_{x} \in \Sigma_{I H}$. Suppose $p_{1}$ agents $B$ mutate by playing $L$ and $p_{2}$ agents $B$ mutate by claiming $x^{\prime}>\widehat{x}_{A}^{D}+\delta$; let $\widetilde{\theta}_{x}$ be the resulting state. Suppose these mutations induce all agents $A$ to play $L$. With positive probability, the process enters the basin of attraction of equilibrium $\theta^{\prime} \in \Sigma_{L H}$ only if updating does not induce all agents $A$ to return to play $H$. By $\widetilde{y}_{L H}$, we denote the expected payoff at $L H$ of agents $A$, which is compatible with the assumption that $\theta_{x}$ is an equilibrium. ${ }^{21}$ Sure enough, at $\widetilde{\theta}_{x}$ all agents $A$ decide to play $L$ if after updating

$$
\frac{N-p_{1}}{N} \widetilde{y}_{L H}>\mu_{H}\left(\widetilde{\theta}_{x}, p_{1}, p_{2}\right)
$$

\footnotetext{
${ }^{21}$ In particular $\widetilde{y}_{L H}$ must not be larger than $\left(V_{H}-x\right)-c$.
} 
where the LHS is the expected payoff by playing $L$ and the RHS is the expected payoff by playing $H$. However, $\mu_{H}\left(\widetilde{\theta}_{x}, p_{1}, p_{2}\right)$ depends on the best demand in an $H H$ match. In particular

$\mu_{H}()=.\left\{\begin{array}{llll}\frac{\left(N-p_{2}-p_{1}\right)\left(V_{H}-x\right)}{N}+\frac{p_{1}\left(V_{M}-\delta\right)}{N}-c & \text { if } & \frac{\left(N-p_{2}-p_{1}\right)\left(V_{H}-x\right)}{N-p_{1}} \geq\left(V_{H}-x^{\prime}\right) \\ \frac{\left(N-p_{1}\right)\left(V_{H}-x^{\prime}\right)}{N}+\frac{p_{1}\left(V_{M}-\delta\right)}{N}-c & \text { if } & \frac{\left(N-p_{2}-p_{1}\right)\left(V_{H}-x\right)}{N-p_{1}}<\left(V_{H}-x^{\prime}\right) .\end{array}\right.$

Given $\widetilde{y}_{L H}$, the minimum number of mutations in population $B$ are obtained by solving two constrained minimization problems ( $M 1$ and $M 2$ ). In both problems, the objective function is $p_{1}+p_{2}$. In $M 1$ (resp. $M 2$ ), we contemplate the case in which the best action at $H H$ is $V_{H}-x^{\prime}$ (resp. $V_{H}-x$ ). Both problems require $p_{1}=0$ as a solution. Moreover, $p_{2}^{M 1}=N\left(\frac{x^{\prime}-x}{V_{H}-x}\right)$ is the solution of $M 1$ for whatever value of $\widetilde{y}_{L H}$, and $p_{2}^{M 2}\left(\widetilde{y}_{L H}\right)=N\left(1-\frac{\widetilde{y}_{L H}+c}{V_{H}-x}\right)$ is the solution of $M 2$. Note that $p_{2}^{M 2}$ depends on $\widetilde{y}_{L H}$. Suppose that $p_{2}^{M 2}\left(\widetilde{y}_{L H}\right)$ agents $B$ claim $x^{\prime}>x$. By updating, all agents $A$ play $L$ so that only profile $L H$ is observed. Since all agents $B$ claim $V_{M}-\delta$ then, after updating, all agents $A$ learn that $\widetilde{y}_{L H}=\delta$. This implies that no agent $A$ has the incentive to play $H$ if

$$
\frac{N-p_{2}^{M 2}\left(\widetilde{y}_{L H}\right)}{N}\left(V_{H}-x\right)-c \leq \delta,
$$

condition weakly satisfied when

$$
\widetilde{y}_{L H}=\delta \text {. }
$$

Therefore, in $M 2$ the minimum number of mutations of agents $B$ needed to enter the basin of attraction of $\theta^{\prime}$ from $\theta_{x}$ is

$$
p_{2}^{M 2}=N\left(1-\frac{\delta+c}{V_{H}-x}\right) .
$$

Since $p_{2}^{M 1}>p_{2}^{M 2}$, the minimum number of mutations involves that: (i) mutating agents only change their demands in the $H H$ profile; (ii) these mutations cause agent $A$ to shift to action $L$ when the best action in match $H H$ continues to be $\left(V_{H}-x\right)$;(iii) all agents $A$ correctly anticipate the distribution occurring at $L H$. Hence:

$$
\bar{r}_{B}(x)=\left\lfloor N\left(1-\frac{\delta+c}{V_{H}-x}\right)\right\rceil .
$$

\section{Proof of Proposition 11}

The proof proceeds along the lines previously used for $\Gamma_{U G}$ with few modifications. We know from Lemma 10 that, starting from $\theta \in \Sigma_{I H}^{D}$, more than one mutation is required in order to enter the basin of attraction of one equilibrium 
which does not belong to $\Sigma_{I H}^{D}$. It is easy to observe that only $\widehat{x}_{B}^{D}-\delta$ and $\widehat{x}_{A}^{D}+\delta$ can be supported respectively as distributional rule under $\Sigma_{C H}^{D}=\Sigma_{H} \backslash \Sigma_{I H}^{D}$

Consider an equilibrium $\theta \in \Sigma_{C H}^{D}$ with $\widehat{x}_{B}^{D}-\delta$ as distributional rule and suppose that a single mutation from $\widehat{x}_{B}^{D}-\delta$ to $x^{\prime}>\widehat{x}_{B}^{D}-\delta$ occurs. Allow all agents $A$ to revise. Since $\left(\widehat{x}_{B}^{D}-\delta\right) \frac{N-1}{N}-c$ is positive ${ }^{22}$ the best action at $H H$ continues to be $\widehat{x}_{B}^{D}-\delta$. However, given that $\left(\widehat{x}_{B}^{D}-\delta\right) \frac{N-1}{N}-c<\delta$, updating will now cause agents $A$ to change investment action and to play $L$. Therefore when $\theta \in \Sigma_{C H}$ one mutation is enough to leave $\Sigma(\theta)$ and enter the basin of attraction of $\theta^{\prime}, \theta^{\prime} \in\left(\Sigma_{H L} \cup \Sigma_{L H}\right)$. Hence, from Lemmas 4 and 5, we can deduce that, for any $\theta \notin \Sigma_{I H}^{D}$, the minimal modified cost for all paths from $\theta$ to $\Sigma_{I H}^{D}$ is equal to one, whatever the number of limit sets the path goes through. Therefore $C R^{*}\left(\Sigma_{I H}^{D}\right)=1$. Since we know from point (a) of Lemma 10 that $R\left(\Sigma_{I H}^{D}\right)>1$, by a direct application of Ellison's result it follows that all stochastically stable states are included in $\Sigma_{I H}^{D}$. Concerning the radius of $\Sigma(\theta)$ for every $\theta \in \Sigma_{I H}^{D}$, Lemma 17 continues to hold but now Lemma 18 replaces Lemma 16. Since for any $x \in\left[\widehat{x}_{B}^{D} ; \widehat{x}_{A}^{D}\right]$ it is always true that

$$
\begin{aligned}
& r_{B}^{+}(x) \leq \bar{r}_{B}(x) \\
& r_{A}^{-}(x) \leq \bar{r}_{A}(x),
\end{aligned}
$$

the proof of Proposition 11 follows immediately by applying Theorem 2 of Ellison (2000).

${ }^{22}$ This follows from (3), (1) and assumption $c>\delta$. 


\section{References}

[1] Alvard, S. M., 2004. The ultimatum game, fairness and cooperation among big game hunters, in Heinrich et al., (2004), 413-435.

[2] Alvard, S. M., Nolin D. A, 2002. Rousseau's whale hunt ?. Current Anthropology $43,533-559$.

[3] Baker, M. J., Swope K., 2005. Sharing, gift-giving and optimal resource use incentives in hunter-gatherer societies. Mimeo, Department of Economics, US Naval Academy.

[4] Bell, D., 1995. On the nature of sharing: beyond the range of methodological individualism. Current Anthropology 36, 826-830

[5] Binmore, K., 1998. The evolution of fairness norms. Rationality and Society $10,275-301$.

[6] Binmore, K., 2007. Economic man or straw man ?. ELSE working paper 262.

[7] Binmore, K., Shaked, A., 2010. Experimental economics: where next ?. Journal of Economic Behavior and Organization 73, 87-100.

[8] Bohem, C., 2004. What makes humans economically distinctive? A threespecies evolutionary comparison and historical analysis. Journal of Bioeconomics $6,109-135$.

[9] Chibnik, M., 2005. Experimental economics in anthropology: a critical assessment. American Ethnologist 32, 198-209.

[10] Dawid, H., MacLeod, B., 2001. Hold up and the evolution of bargaining conventions. European Journal of Economic and Social Systems 15, 139169 .

[11] Dawid, H., MacLeod, B., 2008. Hold-up and the evolution of investment and bargaining norms. Games and Economic Behavior 62, 26-52.

[12] Ellingsen, T., Robles, J., 2002. Does evolution solve the hold-up problem ?. Games and Economic Behavior 39, 28-53.

[13] Ellison, G., 2000. Basin of attraction, long-run stochastic stability, and the speed of step-by-step evolution. Review of Economic Studies, 67, 17-45.

[14] Hackett, S. C., 1993. Incomplete contracting: a laboratory experimental analysis. Economic Inquiry 31, 274-297.

[15] Hackett, S. C., 1994. Is relational exchange possible in the absence of reputation and repeated contact ?. Journal of Law, Economics and Organizations 10, 360-389. 
[16] Hagen E. H., Hammerstein P., 2006. Game theory and human evolution: a critique of some recent interpretations of experimental games. Theoretical Population Biology, 69, 339-348.

[17] Heinrich et al., 2004. Foundations of Human Sociality. Economic Experiments and Ethnographic Evidence from Fifteen Small-Scale Societies. Oxford University Press.

[18] Hill, K., 2002. Altruistic cooperation during foraging by the Ache, and the evolved human predisposition to cooperate. Human Nature 13, 105-128.

[19] Howkes, K., 1992. Sharing and collective action. In: Smith, E., Winterhalder, B. (Eds.). Evolutionary Ecology and Human Behavior, New York: Aldine de Gruyter, 269-300.

[20] Ichikawa, M., 1983. An examination of the hunting-dependent life of the Mbuti Pygmies, eastern Zaire. African Study Monographs 5, 55-76.

[21] Kaplan, H., Hill, K., 1985. Food sharing among the Ache: tests of explanatory hypotheses. Current Anthropology 26, 223-246.

[22] Noldeke, G., Samuelson, L., 1993. An evolutionary analysis of backward and forward induction. Games and Economic Behavior 5, 425-454.

[23] Troger, T., 2002. Why sunk costs matter for bargaining outcomes: an evolutionary approach. Journal of Economic Theory 102, 375-402.

[24] Woodburn, J., 1982. Egalitarian Societies. Man 17, 431-451.

[25] Young, P., 1993. An evolutionary model of bargaining. Journal of Economic Theory 59, 145-168.

[26] Young, P., 1993a. The evolution of conventions. Econometrica 61, 57-84. 


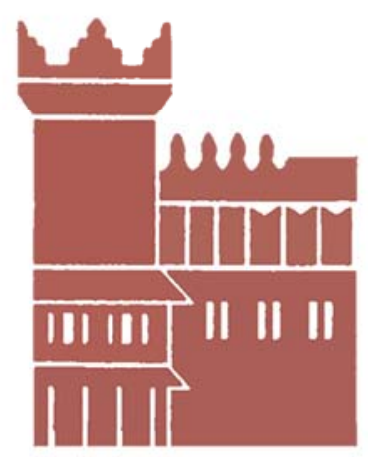

Alma Mater Studiorum - Università di Bologna DEPARTMENT OF ECONOMICS

Strada Maggiore 45

40125 Bologna - Italy

Tel. +39051 2092604

Fax +390512092664

http://www.dse.unibo.it 